Pacific Journal of Mathematic 


\title{
ON CAMERON AND STORVICK'S OPERATOR VALUED FUNCTION SPACE INTEGRAL
}

\author{
KATSUO TAKANO
}

\begin{abstract}
In this paper "the probability density of path space" is introduced by the formula

$$
p_{\lambda}^{\alpha}(t, u)=\frac{1}{2 \pi} \int_{-\infty}^{\infty} \exp \left(-i u \eta-\frac{t}{\lambda}|\eta|^{\alpha}\right) d \eta, \quad(\alpha>0) .
$$

If $\alpha=2$ and $\lambda>0, p_{\lambda}^{\alpha}(t, u)$ is the normal probability density. But if $\alpha>2$ this density can not be considered as a probability density. By this generalization, one can generalize the operator valued function space integral based on the Wiener integral.
\end{abstract}

Introduction. Cameron and Storvick introduced the operator valued function space integral in [1]. Johnson and Skoug [9] developed Cameron and Storvick's theory and improved the results obtained in [1]. To make the arguments in the following sections comprehensible, we will quote the operators $I_{\lambda}^{\sigma}(F)$ and $I_{\lambda}^{\text {sec }}(F)$ from [1], which played an important role in [1], [9]. Let $B[a, b]$ denote the space of real valued functions on an interval $[a, b]$ which are continuous except for a finite number of finite jump discontinuities. Let $F(x)$ be a functional on $B[a, b]$ and $\psi \in L_{2}(-\infty, \infty), \xi \in(-\infty, \infty)$. Then for $\operatorname{Re} \lambda>0$ and any partition $\sigma: a=t_{0}<t_{1}<\cdots<t_{n}=b$, the operator $I_{\lambda}^{\sigma}(F)$ is defined by the formula

$$
\left(I_{\lambda}^{\sigma}(F) \psi\right)(\xi)=\lambda^{n / 2}\left[(2 \pi)^{n}\left(t_{1}-a\right) \cdots\left(t_{n}-t_{n-1}\right)\right]^{-1 / 2} \int_{-\infty}^{\infty}(n) \int_{-\infty}^{\infty} \psi\left(v_{n}\right)
$$

$$
\cdot f_{\sigma}\left(\xi, v_{1}, \cdots, v_{n}\right) \exp \left(-\sum_{j=1}^{n} \frac{\lambda\left(v_{j}-v_{j-1}\right)^{2}}{2\left(t_{j}-t_{j-1}\right)}\right) d v_{1} \cdots d v_{n}
$$

where $v_{0}=\xi, f_{\sigma}\left(\xi, v_{1}, \cdots, v_{n}\right)=F\left[z\left(\sigma, \xi, v_{1}, \cdots, v_{n} \cdot \cdot\right)\right]$,

$$
z\left(\sigma, \xi, v_{1}, \cdots, v_{n}, t\right)=\left\{\begin{array}{cc}
v_{j} & \text { if } \quad t_{j} \leqq t<t_{j+1}, \quad j=0,1, \cdots, n-1, \\
v_{n} & \text { if } t=b,
\end{array}\right.
$$

and where if $n$ is odd we always choose $\lambda^{n / 2}$ with nonnegative real part. Here $\int(n) \int$ means the $n$-fold integral. If $\lambda>0$, by using the Wiener integral, this can be written as 


$$
\left(I_{\lambda}^{\sigma}(F) \psi\right)(\xi)=\int_{C_{0}[a, b]} F\left(\lambda^{-1 / 2} x_{\sigma}+\xi\right) \psi\left(\lambda^{-1 / 2} x(b)+\xi\right) d x
$$

where

$$
x_{\sigma}(t)=\left\{\begin{array}{llc}
x\left(t_{j-1}\right) & \text { if } & t_{j-1} \leqq t<t_{j} \\
& & \\
x(b) & \text { if } & t=b .
\end{array}\right.
$$

As an example important in quantum theory, the functional

$$
F(x)=\exp \left\{\int_{a}^{b} \theta(s, x(s)) d s\right\}
$$

is discussed in [1], [9]. In this case $f_{\sigma}\left(\xi, v_{1}, \cdots, v_{n}\right)$ is given by

$$
\exp \left\{\sum_{j=1}^{n} \int_{t_{j-1}}^{t_{j}} \theta\left(s, v_{j-1}\right) d s\right\}
$$

Let us denote $\max \left\{t_{j}-t_{j-1}\right\}$ by norm $\sigma$ or $\|\sigma\|$. For $\operatorname{Re} \lambda>0$ the operator $I_{\lambda}^{\text {sec }}(F)$ is defined by

$$
I_{\lambda}^{\mathrm{sec}}(F)=w \lim _{\|\sigma\| \rightarrow 0} I_{\lambda}^{\sigma}(F)
$$

where $w$ lim refers to the limit with respect to the weak operator topology. [1] proved that for a class of functionals $F, I_{\lambda}^{\text {sec }}(F)$ exists by using the Wiener integral, and furthermore that $I_{\lambda}^{\text {sec }}(F)$ converges in the weak operator topology as $\lambda=p-i q \rightarrow+0-i q$ for almost all $q \neq 0$. [9] proved that for a class of functionals $F, I_{\lambda}^{\text {sec }}(F)$ exists as the strong operator limit, and furthermore that $I_{\lambda}^{\text {sec }}(F)$ converges in the strong operator topology as $\lambda=p-i q \rightarrow+0-i q$ for all $q \neq 0$ by using the analytic continuation of the Wiener integral. In this paper we introduce the following operator from $L_{2}$ to $L_{2}$ in $\$ 2$ corresponding to $(0.1)$,

$$
\begin{aligned}
\left(\mathscr{F}_{\lambda}^{\alpha, \sigma}(F) \psi\right)(\xi)= & \int_{-\infty}^{\infty} p_{\lambda}^{\alpha}\left(t_{1}-a, v_{1}-\xi\right) d v_{1} \int_{-\infty}^{\infty} p_{\lambda}^{\alpha}\left(t_{2}-t_{1}, v_{2}-v_{1}\right) d v_{2} \mid \\
& \cdots \int_{-\infty}^{\infty} p_{\lambda}^{\alpha}\left(t_{n}-t_{n-1}, v_{n}-v_{n-1}\right) \psi\left(v_{n}\right) f_{\sigma}\left(v_{1}, v_{2}, \cdots, v_{n}\right) d v_{n},
\end{aligned}
$$

for $\operatorname{Re} \lambda \geqq 0, \lambda \neq 0$. We note that if $\operatorname{Re} \lambda>0, p_{\lambda}^{1}(t, u)$ and $p_{\lambda}^{2}(t, u)$ are especially given by 


$$
\begin{aligned}
& p_{\lambda}^{1}(t, u)=\frac{1}{\pi} \frac{t \lambda}{t^{2}+(\lambda u)^{2}}, \\
& p_{\lambda}^{2}(t, u)=\left(\frac{\lambda}{4 \pi t}\right)^{1 / 2} \exp \left(-\frac{\lambda u^{2}}{4 t}\right),
\end{aligned}
$$

respectively.

In general $p_{\lambda}^{\alpha}(t, u)$ is given by the formula

$$
p_{\lambda}^{\alpha}(t, u)=\frac{1}{2 \pi} \int_{-\infty}^{\infty} \exp \left(-i u \eta-\frac{t}{\lambda}|\eta|^{\alpha}\right) d \eta
$$

for $\alpha>0, \quad t>0$ and $\operatorname{Re} \lambda>0$. [1]-[3] and [6]-[10] used only (0.3). Even by this generalization we can also show that for a class of functionals $F, \mathscr{F}_{\lambda}^{\alpha, \sigma}(F)$ converges in the strong operator topology as norm $\sigma \rightarrow 0$. Furthermore we can show that the same integral equation as in [1] [9] holds.

1. Stable density and semigroup. The stable density of exponent $\alpha$ is

$$
p_{1}^{\alpha}(t, u)=\frac{1}{2 \pi} \int_{-\infty}^{\infty} \exp \left(-i u \eta-t|\eta|^{\alpha}\right) d \eta
$$

where $0<\alpha \leqq 2,0<t<\infty$. cf. [12].

It is well-known that (1.1) satisfies the Chapman and Kolmogorov equation, that is, when $\lambda=1$

$$
p_{\lambda}^{\alpha}(t+s, u)=\int_{-\infty}^{\infty} p_{\lambda}^{\alpha}(s, u-y) p_{\lambda}^{\alpha}(t, y) d y
$$

If we consider $p_{\lambda}^{\alpha}(t, u)$ in the operator's sense in the Hilbert space $L_{2}$, (1.2) holds for $\operatorname{Re} \lambda>0$. We shall denote the Fourier transform of $f \in L_{2}$ by $U f$ and the inverse Fourier transform by $U^{*} f$, that is,

$$
\begin{gathered}
(U f)(y)=(2 \pi)^{-1 / 2^{(y)}} \int_{-\infty}^{\infty} \exp (-i y x) f(x) d x, \\
\left(U^{*} f\right)(y)=(2 \pi)^{-1 / 2^{(y)}} \int_{-\infty}^{\infty} \exp (i y x) f(x) d x,
\end{gathered}
$$

where $(y)$ denotes the so-called limit in the mean.

In what follows, let us assume that $\alpha>0$. 
Let

$$
\left(P_{\lambda}^{\alpha}(t) f\right)(y)=U^{*}\left(\exp \left(-\frac{t}{\lambda}|\xi|^{\alpha}\right)(U f)(\xi)\right)(y)
$$

where $f \in L_{2}$ and $\lambda \in D \equiv\{\lambda: \operatorname{Re} \lambda \geqq 0, \lambda \neq 0\}$.

THEOREM 1.1. $\quad\left\{P_{\lambda}^{\alpha}(t): t \geqq 0\right\}$ is a strongly continuous semigroup of contraction operators on $L_{2}$. Furthermore $P_{\lambda}^{\alpha}(t)$ is also strongly continuous with respect to $\lambda$ on $D$.

This follows from the fact that the Fourier transformation is a unitary operator on $L_{2}$.

Lemma 1.1. Let $\operatorname{Re} \lambda>0$ and $t>0$. Then

$p_{\lambda}^{\alpha}(t, \cdot)$ is $L_{2}$-integrable,

$\left|p_{\lambda}^{\alpha}(t, u)\right| \leqq M(\alpha, t, \lambda)<\infty$,

and $p_{\lambda}^{\alpha}(t, u)$ is continuous in $t, \lambda$ and $u$.

Proof. Let $\operatorname{Re} \lambda>0$. Then

$$
\left|p_{\lambda}^{\alpha}(t, u)\right| \leqq \frac{1}{2 \pi} \int_{-\infty}^{\infty}\left|\exp \left(-\frac{t}{\lambda}|\eta|^{\alpha}\right)\right| d \eta
$$

Let $t / \lambda=\delta-i \gamma,(\delta>0)$. We obtain

$$
\begin{aligned}
\left|p_{\lambda}^{\alpha}(t, u)\right| & \leqq \frac{1}{2 \pi} \int_{-\infty}^{\infty} \exp \left(-\delta|\eta|^{\alpha}\right) d \eta=\frac{1}{\pi} \int_{0}^{\infty} \exp \left(-\delta \eta^{\alpha}\right) d \eta \\
& =\frac{1}{\pi} \beta \delta^{-\beta} \Gamma(\beta)<\infty,
\end{aligned}
$$

where $\beta=1 / \alpha$ and $\Gamma(\beta)$ is the gamma function. On using the Dominated Convergence Theorem, we can show that $p_{\lambda}^{\alpha}(t, u)$ is continuous in $t, \lambda$ and $u$. From the above proof, clearly it holds that

$$
(2 \pi)^{-1 / 2} \exp \left(-\frac{t}{\lambda}|\eta|^{\alpha}\right) \in L_{p}, \quad(p=1,2, \cdots) .
$$

We may write $(0.4)$ as

$$
p_{\lambda}^{\alpha}(t, u)=U\left[(2 \pi)^{-1 / 2} \exp \left(-\frac{t}{\lambda}|\cdot|^{\alpha}\right)\right](u) .
$$


Since the Fourier transformation is a unitary operator on $L_{2}, p_{\lambda}^{\alpha}(t, u)$ is $L_{2}$-integrable in $u$.

Lemma 1.2. Let $\operatorname{Re} \lambda>0$ and $t>0$. Then $p_{\lambda}^{\alpha}(t, \cdot)$ is $L_{1^{-}}$ integrable.

Proof. Let

$$
f(\xi)=(2 \pi)^{-1 / 2} \exp \left(-\frac{t}{\lambda}|\xi|^{\alpha}\right)
$$

The derivative of $f(\xi)$ is

$$
f^{\prime}(\xi)=(2 \pi)^{-1 / 2} \exp \left(-\frac{t}{\lambda}|\xi|^{\alpha}\right)\left(-\frac{t}{\lambda}|\xi|^{\alpha-1}\right) \alpha \operatorname{sgn} \xi
$$

where

$$
\operatorname{sgn} \xi=\left\{\begin{array}{rll}
-1 & \text { if } \quad \xi<0 \\
1 & \text { if } \quad \xi>0
\end{array}\right.
$$

Then since $f$ is absolutely continuous on each bounded interval and $f^{\prime}$ is absolutely integrable, it holds that $\left(U f^{\prime}\right)(\xi)=i \xi(U f)(\xi)$. Therefore in order to show that $U f$ is $L_{1}$-integrable, by [13. 12.42. p. 382], it is sufficient to show that $U f^{\prime} \in L_{q}(1,+\infty)$ and $\left(U f^{\prime}\right)(-\cdot) \in L_{q}(1,+\infty)$ since $1 / \xi \in L_{p}(1,+\infty)$, where $1 / p+1 / q=1, p, q>1$. With respect to $f^{\prime}$, it holds that

$$
\int_{-\infty}^{\infty}\left|f^{\prime}(\xi)\right|^{p} d \xi=2(2 \pi)^{-p / 2} \alpha^{p-1}\left|\frac{t}{\lambda}\right|^{p} \int_{0}^{\infty} \tau^{(\beta-1)(1-p)} \exp (-\delta p \tau) d \tau
$$

where $1 / \alpha=\beta$ and $\operatorname{Re}(t / \lambda)=\delta$. Hence $f^{\prime}$ is $L_{p}$-integrable if $(\beta-1)(1-p)>-1$ holds. For a fixed $\alpha$, there always exists a number $p$ in the interval $(1,2]$ such that $(\beta-1)(1-p)>-1$. Therefore we can consider that $f^{\prime}$ is $L_{p}$-integrable for some $p(>1)$. When $f^{\prime}$ is $L_{p}$ integrable, by using [14. Theorem (3.2) p. 254], we obtain that

$$
F(\xi, a)=(2 \pi)^{-1 / 2} \int_{-a}^{a} \exp (-i \xi x) f^{\prime}(x) d x
$$

converges in mean with exponent $q$ as $a \rightarrow \infty$. Since $f^{\prime}$ is $L_{1}$-integrable, $F(\xi, a)$ also converges for all $\xi$ as $a \rightarrow \infty$. Then it follows from [13.12.5 $12.51]$ that the pointwise limit is equal to the limit in mean with exponent 
$q$ for almost all $\xi$. Therefore $U f^{\prime}$ is $L_{q}$-integrable, hence $\left(U f^{\prime}\right)(\xi)$ and $\left(U f^{\prime}\right)(-\xi)$ are in $L_{q}(1,+\infty)$.

Lemma 1.3. Let $\operatorname{Re} \lambda>0$ and $t>0$. Then for $f \in L_{2}$

$$
\int_{-\infty}^{\infty} p_{\lambda}^{\alpha}(t, u-y) f(u) d u
$$

is $L_{2}$-integrable and continuous in $y$.

Proof. Let us put

$$
g(y)=\int_{-\infty}^{\infty} p_{\lambda}^{\alpha}(t, u-y) f(u) d u .
$$

By Lemma 1.1 and the Schwartz inequality, we have

$$
|g(y)-g(y+h)| \leqq\|f\|\left[\int_{-\infty}^{\infty}\left|p_{\lambda}^{\alpha}\left(t, u-y_{1}\right)-p_{\lambda}^{\alpha}(t, u-y-h)\right|^{2} d u\right]^{1 / 2} .
$$

Hence from [13. 19. p. 397], it follows that $g(y)$ is continuous in $y$.

It holds that

$$
\begin{aligned}
\|g\|^{2} & \leqq \int_{-\infty}^{\infty}\left(\int_{-\infty}^{\infty}\left|p_{\lambda}^{\alpha}(t, x-y)\right||f(x)| d x\right)\left(\int_{-\infty}^{\infty}\left|p_{\lambda}^{\alpha}(t, u-y)\right||f(u)| d u\right) d y \\
& =\int_{-\infty}^{\infty} \int_{-\infty}^{\infty} \int_{-\infty}^{\infty}\left|p_{\lambda}^{\alpha}(t, x-y)\right|\left|p_{\lambda}^{\alpha}(t, u-y)\right||f(x)||f(u)| d u d x d y .
\end{aligned}
$$

Let us make a change of variables, $x=x, x-y=z, u-y=v$. Then we have

$$
\frac{\partial(x, y, u)}{\partial(x, v, z)}=1
$$

Hence we obtain by Lemma 1.2 that

$$
\begin{aligned}
\|g\|^{2} & \leqq \int_{-\infty}^{\infty} \int_{-\infty}^{\infty} \int_{-\infty}^{\infty}\left|p_{\lambda}^{\alpha}(t, z)\left\|p_{\lambda}^{\alpha}(t, v)\right\| f(x) \| f(v+x-z)\right| d x d v d z \\
& \leqq\|f\|^{2}\left\|p_{\lambda}^{\alpha}(t, \cdot)\right\|_{1}^{2},
\end{aligned}
$$

where $\|\cdot\|_{1}$ denotes $L_{1}$-norm.

Theorem 1.2. Let $\operatorname{Re} \lambda>0$ and $t>0$. Then for $f \in L_{2}$ 


$$
\left(P_{\lambda}^{\alpha}(t) f\right)(y)=\int_{-\infty}^{\infty} p_{\lambda}^{\alpha}(t, u-y) f(u) d u
$$

Proof. Case I. Let $f \in L_{1} \cap L_{2}$. Then $U f \in L_{2}$. By this and (1.4), we can change the order of integration of (1.3) by the Fubini Theorem, therefore it follows from this fact that

$$
\left(P_{\lambda}^{\alpha}(t) f\right)(y)=\int_{-\infty}^{\infty} p_{\lambda}^{\alpha}(t, u-y) f(u) d u
$$

Case II. Let $f \in L_{2}$. Then we put

$$
f_{n}(x)=\left\{\begin{array}{ccc}
f(x) & \text { if } & |x| \leqq n \\
0 & \text { if } & |x|>n
\end{array}\right.
$$

Since $f_{n} \in L_{1} \cap L_{2}$, it holds that

$$
\left(P_{\lambda}^{\alpha}(t) f_{n}\right)(y)=\int_{-\infty}^{\infty} p_{\lambda}^{\alpha}(t, u-y) f_{n}(u) d u
$$

and

$$
\left\|P_{\lambda}^{\alpha}(t) f-P_{\lambda}^{\alpha}(t) f_{n}\right\| \leqq\left\|f_{n}-f\right\| \rightarrow 0 \quad \text { as } \quad n \rightarrow \infty .
$$

Let us put

$$
g(y)=\int_{-\infty}^{\infty} p_{\lambda}^{\alpha}(t, u-y) f(u) d u
$$

and

$$
g_{n}(y)=\int_{-\infty}^{\infty} p_{\lambda}^{\alpha}(t, u-y) f_{n}(u) d u
$$

We obtain the following by using the inequality (1.6),

$$
\left\|g-g_{n}\right\| \leqq d\left\|f-f_{n}\right\| \rightarrow 0 \quad \text { as } \quad n \rightarrow \infty .
$$

Therefore (1.7) holds.

COROLlaRy 1.1. Let $q$ be a nonzero real number and let

$$
p_{1 q}^{2}(t, x)=\left(\frac{i q}{4 \pi t}\right)^{1 / 2} \exp \left(-\frac{i q}{4 t} x^{2}\right)
$$


Then for $f \in L_{2}$

$$
\left(P_{i q}^{2}(t) f\right)(y)=\int_{-\infty}^{(y)} p_{i q}^{2}(t, u-y) f(u) d u .
$$

Proof. Let $\operatorname{Re} \lambda>0$ and $f \in L_{2}$. We see that

$$
\begin{aligned}
& \left\|\int_{-\infty}^{(\cdot)} p_{i q}^{2}(t, u-\cdot) f(u) d u-\left(P_{i q}^{2}(t) f\right)(\cdot)\right\| \\
& \leqq \\
& \quad\left\|^{(\cdot)} \int_{-\infty}^{\infty} p_{i q}^{2}(t, u-\cdot) f(u) d u-\int_{-\infty}^{\infty} p_{\lambda}^{2}(t, u-\cdot) f(u) d u\right\| \\
& \quad+\left\|\left(P_{\lambda}^{2}(t) f\right)(\cdot)-\left(P_{i q}^{2}(t) f\right)(\cdot)\right\| \rightarrow 0 \quad \text { as } \quad \lambda \rightarrow i q,
\end{aligned}
$$

by Theorem 1.1, Theorem 1.2 and the proof of Theorem in [6. p. 778].

Note 1.1. From the assertion of Theorem 1.2, for convenience, we write $\left(P_{\lambda}^{\alpha}(t) f\right)(y)$ as

$$
\int_{-\infty}^{\infty} p_{\lambda}^{\alpha}(t, u-y) f(u) d u
$$

even if $\lambda=i q$, where $q$ is a nonzero real number. In what follows, we always use the notation (1.8) instead of $P_{\lambda}^{\alpha}(t) f$ for $\lambda \in D$.

Lemma 1.4. Let $t>0$. Then $\left\|p_{\lambda}^{\alpha}(t, \cdot)\right\|$ and $\left\|p_{\lambda}^{\alpha}(t, \cdot)\right\|_{1}$ are continuous functions of $\lambda$ on $C^{+} \equiv\{\lambda: \operatorname{Re} \lambda>0\}$.

Proof. By (1.5) we have

$$
\int_{-\infty}^{\infty}\left|p_{\lambda}^{\alpha}(t, \xi)\right|^{2} d \xi=\frac{1}{2 \pi} \int_{-\infty}^{\infty}\left|\exp \left(-\frac{t}{\lambda}|\eta|^{\alpha}\right)\right|^{2} d \eta
$$

Therefore $\left\|p_{\lambda}^{\alpha}(t, \cdot)\right\|$ is continuous in $\lambda$. From (1.5) and the proof of Lemma 1.2, and using $f$ as defined there we have

$$
\begin{aligned}
\int_{-\infty}^{\infty}\left|p_{\lambda}^{\alpha}(t, \xi)\right| d \xi= & \int_{-1}^{1}|(U f)(\xi)| d \xi+\int_{1}^{\infty} \frac{1}{\xi}\left|\left(U f^{\prime}\right)(\xi)\right| d \xi \\
& +\int_{1}^{\infty} \frac{1}{\xi}\left|\left(U f^{\prime}\right)(-\xi)\right| d \xi
\end{aligned}
$$

The first term is continuous in $\lambda . \quad$ By [14. Theorem (3.2), p. 254] it holds that 


$$
\int_{1}^{\infty}\left|\left(U f^{\prime}\right)(\xi)\right| \xi^{-1} d \xi \leqq\left(\int_{1}^{\infty} \xi^{-p} d \xi\right)^{1 / p} \cdot\left\|U f^{\prime}\right\|_{q} \leqq K\left\|f^{\prime}\right\|_{p}
$$

where let $p$ satisfy the inequalities $(\beta-1)(1-p)>-1$ and $1<p \leqq 2$, and $K$ is constant. By replacing $f$ in the above by

$$
h(\xi, \lambda, \Delta \lambda)=(2 \pi)^{-1 / 2} \exp \left(-\frac{t}{\lambda+\Delta \lambda}|\xi|^{\alpha}\right)-\exp \left(-\frac{t}{\lambda}|\xi|^{\alpha}\right)
$$

we can show that the second term of (1.9) is continuous in $\lambda$. The above argument can be applicable to the third term of (1.9). Therefore the left side of (1.9) is continuous in $\lambda$.

\section{Definition of operator valued function space} integral. Let $C[a, b]$ denote the space of real valued right continuous functions defined on the interval $[a, b]$ and $C_{0}[a, b]$ denote those $x \in C[a, b]$ such that $x(a)=0$. Let $\sigma$ be any partition of $[a, b], \sigma$ : $a=t_{0}<t_{1}<\cdots<t_{n}=b$. For any $x \in C[a, b]$, let $x_{\sigma}(t)=x\left(t_{1}\right)$ if $t_{j-1}<$ $t \leqq t_{j}$, and $x_{\sigma}(a)=x(a)$. Let $F(x)$ be a bounded functional defined on $B[a, b]$. We suppose that $F\left(x_{\sigma}\right)$ has the form

$$
F\left(x_{\sigma}\right)=f_{\sigma}\left(x\left(t_{1}\right), x\left(t_{2}\right), \cdots, x\left(t_{n}\right)\right), \quad(x \in C[a, b])
$$

where $f_{\sigma}\left(v_{1}, v_{2}, \cdots, v_{n}\right)$ is a bounded Borel function on $R^{n}$, and that for each $\lambda \in D$ the operator $\mathscr{F}_{\lambda}^{\alpha, \sigma}(F)$ on $L_{2}$ can be defined by

$$
\left(\underset{\mathscr{F}_{\lambda}^{\alpha, \sigma}}{\alpha,}(F) \psi\right)(\xi)=\int_{-\infty}^{\infty} p_{\lambda}^{\alpha}\left(t_{1}-a, v_{1}-\xi\right) d v_{1} \int_{-\infty}^{\infty} p_{\lambda}^{\alpha}\left(t_{2}-t_{1}, v_{2}-v_{1}\right) d v_{2}
$$

$$
\cdots \int_{-\infty}^{\infty} p_{\lambda}^{\alpha}\left(b-t_{n-1}, v_{n}-v_{n-1}\right) f_{\sigma}\left(v_{1}, v_{2}, \cdots, v_{n}\right) \psi\left(v_{n}\right) d v_{n}
$$

where $\psi \in L_{2}$.

DEFINITION 2.1. If $\mathscr{F}_{\lambda}^{\alpha, \sigma}(F)$ converges in the weak operator topology as norm $\sigma$ tends to 0 for $\lambda \in D$, we denote its limit by $\mathscr{F}_{\lambda}^{\alpha}(F)$ and for the moment we call $\mathscr{F}_{\lambda}^{\alpha}(F)$ the operator valued function space integral of $F$.

We should note from (0.3) that if $\operatorname{Re} \lambda>0,(2.2)$ corresponds to (0.2) except the fact that $f_{\sigma}$ in $(0.2)$ has the variable $v_{0}$. To compare the operator valued function space integral with the Wiener integral, it is convenient to use the following notations; 


$$
\left(\underset{\mathscr{F}_{\lambda}, \sigma}{\alpha, \sigma}(F) \psi\right)(\xi)=\int_{C_{0}[a, b]} F\left(x_{\sigma}+\xi\right) \psi(x(b)+\xi) d p_{\lambda}^{\alpha}(x)
$$

for $(2.2)$ and

$$
\left(\underset{\mathscr{F}_{\lambda}^{\alpha}}{\lambda}(F) \psi\right)(\xi)=\int_{C_{0}[a, b]} F(x+\xi) \psi(x(b)+\xi) d p_{\lambda}^{\alpha}(x) .
$$

LEMMA 2.1. Let $F(x)$ be a factorable functional given by

$$
F(x)=f_{1}\left(x\left(s_{1}\right)\right) f_{2}\left(x\left(s_{2}\right)\right) \cdots f_{m}\left(x\left(s_{m}\right)\right), \quad a<s_{1}<\cdots<s_{m}=b,
$$

where $f_{i}(v)$ are bounded Borel functions. Then for $\lambda \in D, \underset{\lambda}{\mathscr{F}_{\lambda}^{\alpha, \sigma}}(F) \psi$ converges to

$$
\begin{aligned}
\left(\mathscr{F}_{\lambda}^{\alpha}(F) \psi\right)(\xi)= & \int_{-\infty}^{\infty} p_{\lambda}^{\alpha}\left(s_{1}-a, v_{1}-\xi\right) f_{1}\left(v_{1}\right) d v_{1} \ldots \\
& \cdot \int_{-\infty}^{\infty} p_{\lambda}^{\alpha}\left(b-s_{m-1}, v_{m}-v_{m-1}\right) f_{m}\left(v_{m}\right) \psi\left(v_{m}\right) d v_{m}
\end{aligned}
$$

in the norm topology as norm $\sigma \rightarrow 0$, where $\psi \digamma L_{2}$.

Proof. Let $\sigma$ be any partition of $[a, b], \sigma: a=t_{0}<t_{1}<\cdots<t_{n}=$ $b$. Clearly $F\left(x_{\sigma}\right)$ can be expressed as

$$
F\left(x_{\sigma}\right)=f_{1}\left(x\left(t_{r(1)}\right)\right) f_{2}\left(x\left(t_{r(2)}\right)\right) \cdots f_{m}(x(b))
$$

where $t_{r(j)-1}<s_{j} \leqq t_{r(j)}$. Then

$$
\left(\underset{\mathscr{F}_{\lambda}^{\alpha, \sigma}}{\mathscr{N}_{\lambda}}(F) \psi\right)(\xi)=\int_{-\infty}^{\infty} p_{\lambda}^{\alpha}\left(t_{1}-a, v_{1}-\xi\right) d v_{1} \int_{-\infty}^{\infty} p_{\lambda}^{\alpha}\left(t_{2}-t_{1}, v_{2}-v_{1}\right) d v_{2}
$$

$$
\cdots \int_{-\infty}^{\infty} p_{\lambda}^{\alpha}\left(b-t_{n-1}, v_{n}-v_{n-1}\right) f_{1}\left(v_{r(1)}\right) f_{2}\left(v_{r(2)}\right)
$$

$$
\cdots f_{m}\left(v_{n}\right) \psi\left(v_{n}\right) d v_{n}
$$

Here $\mathscr{F}_{\lambda}^{\alpha, \sigma}(F)$ is a well-defined operator on $L_{2}$ for each $\lambda \in D$. By the semigroup property of Theorem 1.1 and Note 1.1, we obtain when norm $\sigma<\operatorname{Min}\left\{s_{i}-s_{i-1}\right\}$

$$
\begin{aligned}
\left(\mathscr{F}_{\lambda}^{\alpha, \sigma}(F) \psi\right)(\xi)= & \int_{-\infty}^{\infty} p_{\lambda}^{\alpha}\left(t_{r(1)}-a, v_{1}-\xi\right) f_{1}\left(v_{1}\right) d v_{1} \cdots \\
& \cdot \int_{-\infty}^{\infty} p_{\lambda}^{\alpha}\left(b-t_{r(m-1)}, v_{m}-v_{m-1}\right) f_{m}\left(v_{m}\right) \psi\left(v_{m}\right) d v_{m} .
\end{aligned}
$$


Since $t_{r(1)}$ converge to $s_{t},(j=1,2, \cdots, m)$ as norm $\sigma \rightarrow 0$, by using the strong continuity of semigroup of Theorem 1.1 and the boundedness of $f_{j}$, it follows that $\mathscr{F}_{\lambda}^{\alpha, \sigma}(F) \psi \rightarrow \mathscr{F}_{\lambda}^{\alpha}(F) \psi$ in the $L_{2}$-norm topology as norm $\sigma \rightarrow 0$ for $\lambda \in D$.

LEMMA 2.2. If $F$ is a functional to which $\mathscr{F}_{\lambda}^{\alpha}$ applies, then $\mathscr{F}_{\lambda}^{\alpha}(F)$ is a linear operator defined on a linear manifold $\mathfrak{M}$ of functions $\psi$. Moreover the operator valued integral $\mathscr{F}_{\lambda}^{\alpha}$ is linear in the sense that if the linear operators $\mathscr{F}_{\lambda}^{\alpha}(F)$ and $\mathscr{F}_{\lambda}^{\alpha}(G)$ are defined on the same manifold $\mathfrak{M}$, then $\mathscr{F}_{\lambda}^{\alpha}\left(c_{1} F+c_{2} G\right)$ is defined on $\mathfrak{M}$ for each pair of complex numbers $c_{1}, c_{2}$, and

$$
\mathscr{F}_{\lambda}^{\alpha}\left(c_{1} F+c_{2} G\right)=c_{1} \mathscr{F}_{\lambda}^{\alpha}(F)+c_{2} \mathscr{F}_{\lambda}^{\alpha}(G)
$$

In particular, if $F$ and $G$ satisfy the hypotheses of Lemma $2.1, \mathscr{F}_{\lambda}^{\alpha}\left(c_{1} F+\right.$ $c_{2} G$ ) maps $L_{2}$ into $L_{2}$ and satisfies (2.5).

Proof. For each fixed $\sigma$, we note from (2.1) that $f_{\sigma}$ depends linearly on $F$, and hence it follows from $(2.2)$ that $\left(\mathscr{F}_{\lambda}^{\alpha, \sigma}(F) \psi\right)(\xi)$ depends linearly on $F$ as well as on $\psi$. Hence the lemma follows from Definition 2.1 and the linearity of the weak limit and Lemma 2.1.

Note 2.1. If $\operatorname{Re} \lambda>0$, by Lemma 1.1, Lemma 1.2 and the Fubini Theorem, we can write (2.3), (2.4) for all $\xi \in R$ as follows

$$
\begin{aligned}
\left(\mathscr{F}_{\lambda}^{\alpha}(F) \psi\right)(\xi)= & \int_{-\infty}^{\infty}(m) \int_{-\infty}^{\infty} f_{1}\left(v_{1}\right) \cdots f_{m}\left(v_{m}\right) \psi\left(v_{m}\right) p_{\lambda}^{\alpha}\left(s_{1}-a, v_{1}-\xi\right) \\
& \cdots p_{\lambda}^{\alpha}\left(b-s_{m-1}, v_{m}-v_{m-1}\right) d v_{1} \cdots d v_{m}, \\
\left(\mathscr{F}_{\lambda}^{\alpha, \sigma}(F) \psi\right)(\xi)= & \int_{-\infty}^{\infty}(n) \int_{-\infty}^{\infty} f_{1}\left(v_{r(1)}\right) \cdots f_{m}\left(v_{n}\right) \psi\left(v_{n}\right) \\
& \cdot p_{\lambda}^{\alpha}\left(t_{1}-a, v_{1}-\xi\right) \cdots p_{\lambda}^{\alpha}\left(b-t_{n-1}, v_{n}-v_{n-1}\right) d v_{1} \cdots d v_{n} .
\end{aligned}
$$

\section{The operator valued function space integral of a} product integral. Let us consider the following functional,

$$
F(x)=\prod_{J=1}^{m} \int_{a}^{b} \theta_{\jmath}(s, x(s)) d s
$$

where

$$
\theta_{l}(s, u) \text { are Borel measurable functions on }[a, b] \times R \text {, and }
$$

$$
\left|\theta_{l}(s, u)\right| \leqq M_{\jmath}<\infty, \quad(j=1,2, \cdots, m) .
$$


LEMMA 3.1. Let $F(x)$ be a functional given by (3.1), (3.2) and (3.3). Then for each $\psi$ in $L_{2}, \mathscr{F}_{\lambda}^{\alpha, \sigma}(F) \psi$ is a strongly continuous function of $\lambda$ on $D$ and analytic in $C^{+}$.

Proof. For a partition $\sigma: t_{0}=a<t_{1}<\cdots<t_{n}=b$ and any $x \in C[a, b]$, we have

$$
F\left(x_{\sigma}\right)=\int_{a}^{b}(m) \int_{a}^{b} \theta_{1}\left(s_{1}, x_{\sigma}\left(s_{1}\right)\right) \cdots \theta_{m}\left(s_{m}, x_{\sigma}\left(s_{m}\right)\right) d s_{1} \cdots d s_{m} .
$$

Since $x_{\sigma}(s)=x\left(t_{j}\right)$ if $t_{j-1}<s \leqq t_{j}$, and $x_{\sigma}(a)=x(a)$, it holds that

$$
\int_{a}^{b} \theta_{j}\left(s, x_{\sigma}(s)\right) d s=\int_{a}^{t_{1}} \theta_{j}\left(s, x\left(t_{1}\right)\right) d s+\cdots+\int_{t_{n-1}}^{t_{n}} \theta_{j}\left(s, x\left(t_{n}\right)\right) d s .
$$

Let us denote

$$
\int_{t_{i-1}}^{t_{i}} \theta_{j}(s, v) d s
$$

by $\phi_{i}^{i}(v)$. Then we have

$$
F\left(x_{\sigma}\right)=\sum_{i(1)=1}^{n} \cdots \sum_{i(m)=1}^{n} \phi_{i(1)}^{1}\left(x\left(t_{i(1)}\right)\right) \cdots \phi_{i(m)}^{m}\left(x\left(t_{i(m)}\right)\right) .
$$

Since $\phi_{i}^{j}(v)$ are bounded Borel measurable functions on $R$, we can define the following operator on $L_{2}$ for each $\lambda \in D$,

$$
\begin{gathered}
\left(K_{\lambda}^{\sigma}(F) \psi\right)(\xi)=\int_{-\infty}^{\infty} p_{\lambda}^{\alpha}\left(t_{1}-a, v_{1}-\xi\right) d v_{1} \int_{-\infty}^{\infty} p_{\lambda}^{\alpha}\left(t_{2}-t_{1}, v_{2}-v_{1}\right) d v_{2} \cdots \\
\cdot \int_{-\infty}^{\infty} p_{\lambda}^{\alpha}\left(t_{n}-t_{n-1}, v_{n}-v_{n-1}\right) \phi_{i(1)}^{1}\left(v_{i(1)}\right) \\
\cdots \phi_{i(m)}^{m}\left(v_{i(m)}\right) \psi\left(v_{n}\right) d v_{n}
\end{gathered}
$$

where $\psi \in L_{2}$. As we have stated in the proof of Lemma 2.2, it holds that

$$
\left(\mathscr{F}_{\lambda}^{\alpha, \sigma}(F) \psi\right)(\xi)=\sum_{i(1)=1}^{n} \cdots \sum_{i(m)=1}^{n}\left(K_{\lambda}^{\sigma}(F) \psi\right)(\xi)
$$

and by the boundedness of $\theta_{j}$, it holds that

$$
\left\|K_{\lambda}^{\sigma}(F) \psi\right\| \leqq M_{1}\left(t_{i(1)}-t_{i(1)-1}\right) \cdots M_{m}\left(t_{i(m)}-t_{i(m)-1}\right)\|\psi\|
$$


By using the boundedness of $\phi_{l}^{\prime}(v)$ and Theorem 1.1, we can show that $K_{\lambda}^{\sigma}(F) \psi$ is strongly continuous in $\lambda$ on $D$. Therefore $\mathscr{F}_{\lambda}^{\alpha, \sigma}(F) \psi$ is a continuous function of $\lambda$ on $D$. Next we wish to show that $K_{\lambda}^{\sigma}(F) \psi$ is an analytic function of $\lambda$ in $C^{+}$for a fixed $\psi \in L_{2}$. Let $g(\lambda)=\left(K_{\lambda}^{\sigma}(F) \psi, \varphi\right)$, $\varphi \in L_{2}$. As we have shown above, since $g(\lambda)$ is a continuous function of $\lambda$ on $D$, we show that

$$
\int_{\Gamma} g(\lambda) d \lambda=0
$$

for triangular path $\Gamma$ in $C^{+}$. Then by Morera's Theorem, $g(\lambda)$ is the analytic function on $C^{+}$. We can consider the ordered integration of $K_{\lambda}^{\sigma}(F) \psi$ as an $n$-fold Lebesgue integral by Lemma 1.1 and Lemma 1.2 since $\lambda$ is in $C^{+}$. It holds that

$$
\int_{\Gamma} g(\lambda) d \lambda=\int_{-\infty}^{\infty} \overline{\varphi(\xi)}\left[\int_{\Gamma}\left(K_{\lambda}^{\sigma}(F) \psi\right)(\xi) d \lambda\right] d \xi
$$

since $\overline{\varphi(\xi)} \cdot\left(K_{\lambda}^{\sigma}(F) \psi\right)(\xi)$ is integrable with respect to $\lambda, \xi$ over $\Gamma \times R$ by the Schwartz inequality and (3.6). Moreover by Lemma 1.1, Lemma 1.2 and Lemma 1.4

$$
\left|\psi\left(v_{n}\right)\right|\left|p_{\lambda}^{\alpha}\left(t_{1}-a, v_{1}-\xi\right) \cdots p_{\lambda}^{\alpha}\left(t_{n}-t_{n-1}, v_{n}-v_{n-1}\right)\right|
$$

is integrable with respect to $v_{1}, \cdots, v_{n}, \lambda$ over $R^{n} \times \Gamma$, therefore by using the Fubini Theorem,

$$
\begin{array}{r}
\int_{\Gamma}\left(K_{\lambda}^{\sigma}(F) \psi\right) d \lambda=\int_{-\infty}^{\infty}(n) \int_{-\infty}^{\infty}\left[\int_{\Gamma} p_{\lambda}^{\alpha}\left(t_{1}-a, v_{1}-\xi\right)\right. \\
\left.\cdots p_{\lambda}^{\alpha}\left(t_{n}-t_{n-1}, v_{n}-v_{n-1}\right) d \lambda\right] \\
\cdot \phi_{l(1)}^{1}\left(v_{l(1)}\right): \cdots \phi_{l(m)}^{m}\left(v_{l(m)}\right) \psi\left(v_{n}\right) d v_{1} \cdots d v_{n} .
\end{array}
$$

By (0.4) it holds that

$$
\int_{\Gamma} \prod_{j=1}^{n} p_{\lambda}^{\alpha}\left(t_{\jmath}-t_{j-1}, v_{\jmath}-v_{j-1}\right) d \lambda=0
$$

Therefore we obtain

$$
\int_{\Gamma} g(\lambda) d \lambda=0
$$


Let us put

$$
S(\tau)=\left\{\left(s_{1}, \cdots, s_{m}\right) \in(a, b)^{m}: a<s_{\tau(1)}<\cdots<s_{\tau(m)}<b\right\} .
$$

Here $\tau$ means a permutation of $\{1,2, \cdots, m\}$ and $\left(s_{1}, \cdots, s_{m}\right) \in S(\tau)$ means that $\left(s_{1}, \cdots, s_{m}\right)$ satisfies the order relation $a<s_{\tau(1)}<\cdots<s_{\tau(m)}$ $<b$.

THEOREM 3.1. Let $F(x)$ be a functional given by (3.1), (3.2) and (3.3). Then for $\lambda \in D, \underset{\mathscr{F}_{\lambda}^{\alpha, \sigma}}{(F) \psi \text { converges to }}$

$$
\begin{aligned}
\left(\mathscr{F}_{\lambda}^{\alpha}(F)\right. & \psi(\xi) \\
= & \sum_{\tau}(B) \int_{S(\tau)}(m) \int\left[\int_{-\infty}^{\infty} p_{\lambda}^{\alpha}\left(s_{\tau(1)}-a, v_{1}-\xi\right) \theta_{\tau(1)}\left(s_{\tau(1)}, v_{1}\right) d v_{1}\right. \\
& \cdots \int_{-\infty}^{\infty} p_{\lambda}^{\alpha}\left(s_{\tau(m)}-s_{\tau(m-1)}, v_{m}-v_{m-1}\right) \theta_{\tau(m)}\left(s_{\tau(m)}, v_{m}\right) d v_{m} \\
& \left.\cdot \int_{-\infty}^{\infty} p_{\lambda}^{\alpha}\left(b-s_{\tau(m)}, v_{m+1}-v_{m}\right) \psi\left(v_{m+1}\right) d v_{m+1}\right] d s_{1} \cdots d s_{m}
\end{aligned}
$$

in the norm topology as norm $\sigma \rightarrow 0$, where $\psi \in L_{2}$ and the sum is taken over all $m$ ! permutations $\tau$ of $\{1,2, \cdots, m\}$ and (B) denotes the Bochner integral with respect to Lebesgue measure on $S(\tau)$. Furthermore

$$
\left\|\mathscr{F}_{\lambda}^{\alpha}(F)\right\| \leqq(b-a)^{m} M_{1} M_{2} \cdots M_{m} .
$$

Proof. Let $\lambda$ be in $C^{+}$. For all $\xi \in R$, (3.7) is integrable with respect to $v_{1}, \cdots, v_{n}$ over $R^{n}$. By this fact, we can change the order of integration of $K_{\lambda}^{\sigma}(F) \psi$ by the Fubini Theorem, hence we obtain

$$
\begin{aligned}
& \left(K_{\lambda}^{\sigma}(F) \psi\right)(\xi) \\
& \quad=\int_{t_{1}(1)-1}^{t_{t_{(1)}}}(m) \int_{t_{i(m)-1}}^{t_{i_{(m)}}}\left(H_{\lambda}^{\sigma}\left(s_{1}, \cdots, s_{m}\right) \psi\right)(\xi) d s_{1} \cdots d s_{m},
\end{aligned}
$$

where

$$
\begin{gathered}
\left(H_{\lambda}^{\sigma}\left(s_{1}, \cdots, s_{m}\right) \psi\right)(\xi)=\int_{-\infty}^{\infty} p_{\lambda}^{\alpha}\left(t_{1}-a, v_{1}-\xi\right) d v_{1} \int_{-\infty}^{\infty} p_{\lambda}^{\alpha}\left(t_{2}-t_{1}, v_{2}-v_{1}\right) d v_{2} \\
\cdots \int_{-\infty}^{\infty} p_{\lambda}^{\alpha}\left(t_{n}-t_{n-1}, v_{n}-v_{n-1}\right) \theta_{1}\left(s_{1}, v_{i(1)}\right) \cdots \theta_{m}\left(s_{m}, v_{i(m)}\right) \psi\left(v_{n}\right) d v_{n} .
\end{gathered}
$$


From (3.5) and (3.10) we see that

$\left(\mathscr{F}_{\lambda}^{\alpha}, \sigma(F) \psi(\xi)\right.$

$$
\begin{aligned}
= & \int_{a}^{b}(m) \int_{a}^{b} d s_{1} \cdots d s_{m}\left[\int_{-\infty}^{\infty} p_{\lambda}^{\alpha}\left(t_{1}-a, v_{1}-\xi\right) d v_{1}\right. \\
& \cdot \int_{-\infty}^{\infty} p_{\lambda}^{\alpha}\left(t_{2}-t_{1}, v_{2}-v_{1}\right) d v_{2}
\end{aligned}
$$

$$
\begin{aligned}
& \left.\cdots \int_{-\infty}^{\infty} p_{\lambda}^{\alpha}\left(t_{n}-t_{n-1}, v_{n}-v_{n-1}\right) \prod_{j=1}^{m} \theta_{j}\left(s_{J}, V_{\sigma}\left(s_{j}\right)\right) \psi\left(v_{n}\right) d v_{n}\right] \\
= & \sum_{\tau} \int_{S(\tau)}(m) \int\left[\int_{-\infty}^{\infty} p_{\lambda}^{\alpha}\left(t_{1}-a, v_{1}-\xi\right) d v_{1} \int_{-\infty}^{\infty} p_{\lambda}^{\alpha}\left(t_{2}-t_{1}, v_{2}-v_{1}\right) d v_{2}\right. \\
& \left.\cdots \int_{-\infty}^{\infty} p_{\lambda}^{\alpha}\left(t_{n}-t_{n-1}, v_{n}-v_{n-1}\right) \prod_{j=1}^{m} \theta_{J}\left(s_{J}, V_{\sigma}\left(s_{j}\right)\right) \psi\left(v_{n}\right) d v_{n}\right] d s_{1} \cdots d s_{m},
\end{aligned}
$$

where $V_{\sigma}(s)=v_{i}$ if $t_{i-1}<s \leqq t_{i}$.

By rearranging the product $\theta_{1}\left(s_{1}, V_{\sigma}\left(s_{1}\right)\right) \cdots \theta_{m}\left(s_{m}, V_{\sigma}\left(s_{m}\right)\right)$ of the last member of (3.11) as

$$
\theta_{\tau(1)}\left(s_{\tau(1)}, V_{\sigma}\left(s_{\tau(1)}\right)\right) \cdot \theta_{\tau(2)}\left(s_{\tau(2)}, V_{\sigma}\left(s_{\tau(2)}\right)\right) \cdots \theta_{\tau(m)}\left(s_{\tau(m)}, V_{\sigma}\left(s_{\tau(m)}\right)\right)
$$

since $\left(s_{1}, \cdots, s_{m}\right) \in S(\tau),(3.11)$ can be expressed in the following form,

$$
\begin{aligned}
& \left(\mathscr{F}_{\lambda}^{\alpha, \sigma}(F) \psi\right)(\xi) \\
& \quad=\sum_{\tau} \int_{S(\tau)}(m) \int\left(H_{\lambda, \tau}^{\sigma}\left(s_{1}, \cdots, s_{m}\right) \psi\right)(\xi) d s_{1} \cdots d s_{m}
\end{aligned}
$$

where

$$
\begin{aligned}
& \left(H_{\lambda, \tau}^{\sigma}\left(s_{1}, \cdots, s_{m}\right) \psi\right)(\xi) \\
& \equiv \int_{-\infty}^{\infty} p_{\lambda}^{\alpha}\left(t_{1}-a, v_{1}-\xi\right) d v_{1} \int_{-\infty}^{\infty} p_{\lambda}^{\alpha}\left(t_{2}-t_{1}, v_{2}-v_{1}\right) d v_{2} \cdots \\
& \quad \cdot \int_{-\infty}^{\infty} p_{\lambda}^{\alpha}\left(t_{n}-t_{n-1}, v_{n}-v_{n-1}\right) \theta_{\tau(1)}\left(s_{\tau(1)}, V_{\sigma}\left(s_{\tau(1)}\right)\right) \cdots \\
& \quad \cdot \theta_{\tau(m)}\left(s_{\tau(m)}, V_{\sigma}\left(s_{\tau(m)}\right)\right) \psi\left(v_{n}\right) d v_{n},\left(s_{1}, \cdots, s_{m}\right) \in S(\tau) .
\end{aligned}
$$

Now we prove that (3.13) is Bochner integrable over $S(\tau)$. In order to show this, it suffices to show that 
(1) $H_{\lambda, \tau}^{\sigma}\left(s_{1}, \cdots, s_{m}\right) \psi$ is strongly measurable in $\left(s_{1}, \cdots, s_{m}\right)$ on $S(\tau)$,

(2) $\int_{S(\tau)}(m) \int\left\|H_{\lambda, \tau}^{\sigma}\left(s_{1}, \cdots, s_{m}\right) \psi\right\| d s_{1} \cdots d s_{m}<\infty$.

Clearly it follows from Theorem 1.1 and (3.3) that

(a) $\left\|H_{\lambda, \tau}^{\sigma}\left(s_{1}, \cdots, s_{m}\right) \psi\right\| \leqq M_{1} \cdots M_{m}\|\psi\|$,

for almost all $\left(s_{1}, \cdots, s_{m}\right) \in S(\tau)$.

In order to prove (1), it suffices to prove by [5. Corollary 2. p. 73] that $H_{\lambda, \tau}^{\sigma}\left(s_{1}, \cdots, s_{m}\right) \psi$ is weakly measurable on $S(\tau)$, that is $\left(H_{\lambda, \tau}^{\sigma}\left(s_{1}, \cdots, s_{m}\right) \psi, \varphi\right)$ is measurable in $\left(s_{1}, \cdots, s_{m}\right)$ for $\varphi \in L_{2}$. If $\varphi \in$ $L_{1} \cap L_{2}$, from Lemma 1.1, Lemma 1.2 and the boundedness of $\theta_{l}$,

$$
\begin{gathered}
\overline{\varphi(\xi)}\left[\prod_{j=1}^{m} \theta_{\tau(y)}\left(s_{\tau(j)}, V_{\sigma}\left(s_{\tau(j)}\right)\right)\right] \psi\left(v_{n}\right) p_{\lambda}^{\alpha}\left(t_{1}-a, v_{1}-\xi\right) \\
\cdots p_{\lambda}^{\alpha}\left(t_{n}-t_{n-1}, v_{n}-v_{n-1}\right)
\end{gathered}
$$

is integrable with respect to the variables $s_{1}, \cdots, s_{m}, \xi, v_{1}, \cdots, v_{n}$ over $S(\tau) \times R^{n+1}$. Therefore it follows from the Fubini Theorem that $\left(H_{\lambda, \tau}^{\sigma}\left(s_{1}, \cdots, s_{m}\right) \psi, \varphi\right)$ is measurable in $\left(s_{1}, \cdots, s_{m}\right)$ on $S(\tau)$. If $\varphi \in L_{2}$, let us put

$$
\varphi_{N}(x)=\left\{\begin{array}{ccc}
\varphi(x) & \text { if } & |x| \leqq N \\
0 & \text { if } & |x|>N
\end{array}\right.
$$

Then since $\left\|\varphi_{N}-\varphi\right\| \rightarrow 0$ as $N \rightarrow \infty$, it holds by (a) that for almost all $\left(s_{1}, \cdots, s_{m}\right) \in S(\tau)$,

$$
\left(H_{\lambda, \tau}^{\sigma}\left(s_{1}, \cdots, s_{m}\right) \psi, \varphi_{N}\right) \rightarrow\left(H_{\lambda, \tau}^{\sigma}\left(s_{1}, \cdots, s_{m}\right) \psi, \varphi\right) \quad \text { as } \quad N \rightarrow \infty .
$$

By the fact that $\left(H_{\lambda, \tau}^{\sigma}\left(s_{1}, \cdots, s_{m}\right) \psi, \varphi_{N}\right)$ is measurable in $\left(s_{1}, \cdots, s_{m}\right)$, $\left(H_{\lambda, \tau}^{\sigma}\left(s_{1}, \cdots, s_{m}\right) \psi, \varphi\right)$ is also measurable with respect to the variables $s_{1}, \cdots, s_{m}$ on $S(\tau)$. Furthermore $\left\|H_{\lambda, \tau}^{\sigma}\left(s_{1}, \cdots, s_{m}\right) \psi\right\|$ is measurable on $S(\tau)$ by [5. Theorem 3.5.2] since $L_{2}$ is separable. Therefore by (a), (2) holds. Hence we see that

(b) $H_{\lambda, \tau}^{\sigma}\left(s_{1}, \cdots, s_{m}\right) \psi$ is Bochner integrable over $S(\tau)$ if $\lambda \in C^{+}$.

Next we wish to prove that $H_{\lambda, \tau}^{\sigma}\left(s_{1}, \cdots, s_{m}\right) \psi$ is also Bochner integrable over $S(\tau)$ even if $\lambda=-i q(q$ is a nonzero real number). Here we should note that for $\lambda=-i q$, (3.13) is well-defined for almost all $\left(s_{1}, \cdots, s_{m}\right) \in S(\tau)$ by the boundedness of $\theta_{j}$. In order to 
prove the Bochner integrability, by (a), (b) and [5. Theorem 3.7.9] it suffices to prove that for almost all $\left(s_{1}, \cdots, s_{m}\right) \in S(\tau)$,

$$
\left\|H_{\lambda, \tau}^{\sigma}\left(s_{1}, \cdots, s_{m}\right) \psi-H_{-i q, \tau}^{\sigma}\left(s_{1}, \cdots, s_{m}\right) \psi\right\| \rightarrow 0
$$

as $\lambda \rightarrow-i q$. By using the boundedness of $\theta_{\text {, }}$ and Theorem 1.1, we can see that (3.15) holds for almost all $\left(s_{1}, \cdots, s_{m}\right) \in S(\tau)$. Hence we have

(c) $H_{-i q, \tau}^{\sigma}\left(s_{1}, \cdots, s_{m}\right) \psi$ is Bochner integrable over $S(\tau)$,

(d) $\left\|H_{-q q, \tau}^{\sigma}\left(s_{1}, \cdots, s_{m}\right) \psi\right\| \leqq M_{1} \cdots M_{m}\|\psi\|$, for almost all $\left(s_{1}, \cdots\right.$, $\left.s_{m}\right) \in S(\tau)$.

Furthermore, by using the Dominated Convergence Theorem [5. Theorem 3.7.9] we obtain

$$
\begin{aligned}
& \lim _{\lambda \rightarrow-i q}(B) \int_{S(\tau)}(m) \int H_{\lambda, \tau}^{\sigma}\left(s_{1}, \cdots, s_{m}\right) \psi d s_{1} \cdots d s_{m} \\
& \quad=(B) \int_{S(\tau)}(m) \int H_{-\imath q, \tau}^{\sigma}\left(s_{1}, \cdots, s_{m}\right) \psi d s_{1} \cdots d s_{m} .
\end{aligned}
$$

Let $\varphi \in L_{2}$ and $\lambda \in C^{+}$. Then $\left|\overline{\varphi(\xi)}\left(H_{\lambda, \tau}^{\sigma}\left(s_{1}, \cdots, s_{m}\right) \psi\right)(\xi)\right|$ is integrable over $S(\tau) \times R$ by (a). Hence by using the Fubini Theorem and [5. Theorem 3.7.12 and the remark following], we have

$$
\begin{aligned}
& \int_{-\infty}^{\infty} \overline{\varphi(\xi)}\left[\int_{S(\tau)}(m) \int\left(H_{\lambda, \tau}^{\sigma}\left(s_{1}, \cdots, s_{m}\right) \psi\right)(\xi) d s_{1} \cdots d s_{m}\right] d \xi \\
& \quad=\int_{-\infty}^{\infty} \overline{\varphi(\xi)}\left[(B) \int_{S(\tau)}(m) \int\left(H_{\lambda, \tau}^{\sigma}\left(s_{1}, \cdots, s_{m}\right) \psi\right)(\xi) d s_{1} \cdots d s_{m}\right] d \xi
\end{aligned}
$$

From this fact, it follows that for $\lambda \in C^{+}$

$$
\begin{aligned}
\int_{S(\tau)}(m) \int H_{\lambda, \tau}^{\sigma}\left(s_{1}, \cdots, s_{m}\right) \psi d s_{1} \cdots d s_{m} \\
=(B) \int_{S(\tau)}(m) \int H_{\lambda, \tau}^{\sigma}\left(s_{1}, \cdots, s_{m}\right) \psi d s_{1} \cdots d s_{m} .
\end{aligned}
$$

From (3.12) and (3.17) we have

$$
\underset{\mathscr{F}_{\lambda}}{\alpha, \sigma}(F) \psi=\sum_{\tau}(B) \int_{S(\tau)}(m) \int H_{\lambda, \tau}^{\sigma}\left(s_{1}, \cdots, s_{m}\right) \psi d s_{1} \cdots d s_{m}
$$


for $\lambda \in C^{+}$. From this equality, by using (3.16) and Lemma 3.1, we obtain

(3.18) $\underset{\mathscr{F}_{\lambda}^{\alpha, \sigma}}{\mathscr{N}^{\alpha}}(F) \psi=\sum_{\tau}(B) \int_{S(\tau)}(m) \int H_{\lambda, \tau}^{\sigma}\left(s_{1}, \cdots, s_{m}\right) \psi d s_{1} \cdots d s_{m}$

for every $\lambda \in D$.

Now we show that (3.18) converges to (3.8) in the $L_{2}$-norm topology as norm $\sigma \rightarrow 0$. For almost all $\left(s_{1}, \cdots, s_{m}\right) \in S(\tau)$, it follows from (3.13) that

$$
\begin{aligned}
& \left(H_{\lambda, \tau}^{\sigma}\left(s_{1}, \cdots, s_{m}\right) \psi\right)(\xi)=\int_{-\infty}^{\infty} p_{\lambda}^{\alpha}\left(t_{1}-a, v_{1}-\xi\right) d v_{1} \cdots \\
& \quad \cdot \int_{-\infty}^{\infty} p_{\lambda}^{\alpha}\left(t_{n}-t_{n-1}, v_{n}-v_{n-1}\right) \theta_{\tau(1)}\left(s_{\tau(1)}, v_{r(1)}\right) \cdots \\
& \quad \cdot \theta_{\tau(m)}\left(s_{\tau(m)}, v_{r(m)}\right) \psi\left(v_{n}\right) d v_{n},
\end{aligned}
$$

where let $t_{r()-1}<s_{\tau())} \leqq t_{r())}$. By the same argument as in the proof of Lemma 2.1, for almost all $\left(s_{1}, \cdots, s_{m}\right) \in S(\tau)$, (3.19) converges to

$$
\begin{aligned}
& \left.I_{\tau}\left(s_{1}, \cdots, s_{m}\right) \psi\right)(\xi) \\
& \equiv \int_{-\infty}^{\infty} p_{\lambda}^{\alpha}\left(s_{\tau(1)}-a, v_{1}-\xi\right) \theta_{\tau(1)}\left(s_{\tau(1)}, v_{1}\right) d v_{1} \cdots \\
& \quad \cdot \int_{-\infty}^{\infty} p_{\lambda}^{\alpha}\left(s_{\tau(m)}-s_{\tau(m-1)}, v_{m}-v_{m-1}\right) \theta_{\tau(m)}\left(s_{\tau(m)}, v_{m}\right) d v_{m} \\
& \quad \cdot \int_{-\infty}^{\infty} p_{\lambda}^{\alpha}\left(b-s_{\tau(m)}, v_{m+1}-v_{m}\right) \psi\left(v_{m+1}\right) d v_{m+1}
\end{aligned}
$$

in the norm topology as norm $\sigma \rightarrow 0$. From this fact and from (a), (b), (c), (d) and from [5. Theorem 3.7.9], it follows that

(e) $\left\|I_{\tau}\left(s_{1}, \cdots, s_{m}\right) \psi\right\| \leqq M_{1} \cdots M_{m}\|\psi\|$,

for almost all $\left(s_{1}, \cdots, s_{m}\right) \in S(\tau)$,

(f) $I_{\tau}\left(s_{1}, \cdots, s_{m}\right) \psi$ is also Bochner integrable over $S(\tau)$, furthermore

$$
\underset{\mathscr{F}_{\lambda}^{\alpha, \sigma}}{\alpha, F} \psi \psi \rightarrow \sum_{\tau}(B) \int_{S(\tau)}(m) \int I_{\tau}\left(s_{1}, \cdots, s_{m}\right) \psi d s_{1} \cdots d s_{m}
$$

in the norm topology as norm $\sigma \rightarrow 0$ for each $\lambda \in D$. 
Now we prove (3.9). From (e) and [5. Theorem 3.7.6], it follows that

$$
\begin{aligned}
\left\|\mathscr{F}_{\lambda}^{\alpha}(F) \psi\right\| & \leqq \sum_{\tau} \int_{S(\tau)}(m) \int\left\|I_{\tau}\left(s_{1}, \cdots, s_{m}\right) \psi\right\| d s_{1} \cdots d s_{m} \\
& =\sum_{\tau} \frac{(b-a)^{m}}{m !} M_{1} \cdots M_{m}\|\psi\|=(b-a)^{m} M_{1} \cdots M_{m}\|\psi\| .
\end{aligned}
$$

Therefore we have

$$
\left\|\mathscr{F}_{\lambda}^{\alpha}(F)\right\| \leqq(b-a)^{m} M_{1} \cdots M_{n} .
$$

COROllary 3.1. Let $\theta(t, u)=\theta_{1}(t, u)=\cdots=\theta_{m}(t, u)$ in Theorem 3.1. Then for $\lambda \in D$

$$
\begin{aligned}
& \left(\mathscr{F}_{\lambda}^{\alpha}(F) \psi\right)(\xi) \\
& \quad=m !(B) \int_{S_{m}(a, b)}(m) \int\left[\int_{-\infty}^{\infty} p_{\lambda}^{\alpha}\left(s_{1}-a, v_{1}-\xi\right) \theta\left(s_{1}, v_{1}\right) d v_{1}\right.
\end{aligned}
$$

$$
\begin{aligned}
& \cdots \int_{-\infty}^{\infty} p_{\lambda}^{\alpha}\left(s_{m}-s_{m-1}, v_{m}-v_{m-1}\right) \theta\left(s_{m}, v_{m}\right) d v_{m} \\
& \left.\cdot \int_{-\infty}^{\infty} p_{\lambda}^{\alpha}\left(b-s_{m}, v_{m+1}-v_{m}\right) \psi\left(v_{m+1}\right) d v_{m+1}\right] d s_{1} \cdots d s_{m},
\end{aligned}
$$

where

$$
S_{m}(a, b)=\left\{\left(s_{1}, \cdots, s_{m}\right) \in(a, b)^{m}: a<s_{1}<s_{2}<\cdots s_{m}<b\right\}
$$

and $\psi \in L_{2}$.

Note 3.1. Let us denote the right side of (3.21) by $m ! \mathscr{J}_{\lambda}^{\alpha}(F) \psi$. For convenience, we use a notation $\mathscr{J}_{\lambda}^{\alpha}(F)$ in place of $\mathscr{F}_{\lambda}^{\alpha}(F)$ when $F(x) \equiv 1$.

4. The operator valued function space integral of $F(x)=\sum_{m=1}^{\infty} F_{m}(x)$.

Let us denote the set of all the functionals satisfying (3.1), (3.2) and (3.3) by $A_{0}$ and let us introduce the quantity

$$
N_{0}(F)=(b-a)^{m} M_{1} \cdots M_{m},
$$

where let $F$ satisfy (3.1), (3.2) and (3.3). 
Let

$$
A \equiv\left\{F(x)=\sum_{m=1}^{\infty} F_{m}(x): F_{m} \in A_{0} \quad \text { and } \quad \sum_{m=1}^{\infty} N_{0}\left(F_{m}\right)<\infty\right\}
$$

THEOREM 4.1. Let $F$ be in $A$ and let $F(x)=\sum_{m=1}^{\infty} F_{m}(x)$. Then for $\lambda \in D, \underset{\lambda}{\mathscr{F}_{\lambda}^{\alpha, \sigma}}(F) \psi$ converges to $\mathscr{F}_{\lambda}^{\alpha}(F) \psi=\sum_{m=1}^{\infty} \mathscr{F}_{\lambda}^{\alpha}\left(F_{m}\right) \psi$ in the norm topology as norm $\sigma \rightarrow 0$, where $\psi \in L_{2}$.

Furthermore

$$
\left\|\mathscr{F}_{\lambda}^{\alpha}(F) \psi\right\| \leqq \sum_{m=1}^{\infty} N_{0}\left(F_{m}\right)\|\psi\| .
$$

Note 4.1. We do not assume that $F$ determines $F_{1}, F_{2}, \cdots$ uniquely or that $F_{m}$ determines $N_{0}\left(F_{m}\right)$ uniquely; but we assert that (4.2) holds for any choice of $F_{1}, F_{2}, \cdots$ whose sum is $F$, where each $F_{m}$ satisfies (3.1), (3.2), (3.3), (4.1) with the $m$ in those equations corresponding to the subscript of $F_{m}$.

Proof. Let

$$
F_{m}(x)=\prod_{j=1}^{m} \int_{a}^{b} \theta_{j}^{m}(s, x(s)) d s
$$

where each $\theta_{j}^{m}$ is bounded and Borel measurable in $[a, b] \times R$; let $\sigma$ be any partition $a=t_{0}<t_{1}<\cdots<t_{n}=b$; and let

$$
\phi_{i}^{m, j}(v)=\int_{t_{i-1}}^{t_{i}} \theta_{j}^{m}(s, v) d s
$$

Then we have formally that

$$
\begin{aligned}
\left(\underset{\lambda}{\mathscr{F}_{\lambda}^{\alpha, \sigma}}(F) \psi\right)(\xi)= & \int_{-\infty}^{\infty} p_{\lambda}^{\alpha}\left(t_{1}-a, v_{1}-\xi\right) d v_{1} \cdots \int_{-\infty}^{\infty} p_{\lambda}^{\alpha}\left(t_{n}-t_{n-1}, v_{n}-v_{n-1}\right) \\
& \cdot \psi\left(v_{n}\right) \sum_{m=1}^{\infty} F_{m}\left(V_{\sigma}\right) d v_{n}
\end{aligned}
$$

where $\psi \in L_{2}$ and by (3.4)

$$
F_{m}\left(V_{\sigma}\right)=\sum_{i(1)=1}^{n} \cdots \sum_{i(m)=1}^{n} \phi_{i(1)}^{m, 1}\left(v_{i(1)}\right) \cdots \phi_{i(m)}^{m, m}\left(v_{i(m)}\right)
$$

If necessary, by multiplying by additional functions which are identically 1 , we can write $F_{m}\left(V_{\sigma}\right)$ as 


$$
F_{m}\left(V_{\sigma}\right)
$$

$$
=\sum_{i_{1}=1}^{n} \cdots \sum_{l_{m}=1}^{n} \varphi_{1}^{m}\left(i_{1}, \cdots, i_{m}: v_{1}\right) \cdots \varphi_{n}^{m}\left(i_{1}, \cdots, i_{m}: v_{n}\right),
$$

where notation $i_{j}$ is made use of instead of $i(j)$.

From the fact that $F_{m}(x)$ is in $A_{0}$, it is clear that for almost all $v_{1}, \cdots, v_{n}$

$$
\begin{aligned}
& \sum_{i_{1}=1}^{n} \cdots \sum_{l_{m}=1}^{n}\left|\varphi_{1}^{m}\left(i_{1}, \cdots, i_{m}: v_{1}\right) \cdots \varphi_{n}^{m}\left(i_{1}, \cdots, i_{m}: v_{n}\right)\right| \\
& \leqq N_{0}\left(F_{m}\right), \quad(m=1,2, \cdots) .
\end{aligned}
$$

From (4.4), we have for almost all $v_{1}, \cdots, v_{n}$,

$$
\begin{aligned}
& \sum_{m=1}^{\infty}\left(\sum_{\iota_{1}=1}^{n} \cdots \sum_{l_{m}=1}^{n}\left|\varphi_{1}^{m}\left(i_{1}, \cdots, i_{m}: v_{1}\right) \cdots \varphi_{n}^{m}\left(i_{1}, \cdots, i_{m}: v_{n}\right)\right|\right) \\
& \leqq \sum_{m=1}^{\infty} N_{0}\left(F_{m}\right)<\infty .
\end{aligned}
$$

Now we will prove that

$$
\int_{-\infty}^{\infty} p_{\lambda}^{\alpha}\left(b-t_{n-1}, v_{n}-v_{n-1}\right) \psi\left(v_{n}\right) \sum_{m=1}^{\infty} F_{m}\left(V_{\sigma}\right) d v_{n}
$$

is $L_{2}$-integrable with respect to $v_{n-1}$ for almost all $v_{1}, \cdots, v_{n-2}$. By Note $1: 1$ and (4.3), (4.6) is

$$
U_{v_{n}}^{*}\left\{\operatorname { e x p } ( - \frac { b - t _ { n - 1 } } { \lambda } | v _ { n } | ^ { \alpha } ) U _ { \eta } \left[\psi(\eta) \sum_{m=1}^{\infty} \sum_{i_{1}=1}^{n} \cdots \sum_{l_{m}=1}^{n} \varphi_{1}^{m}\left(i_{1}, \cdots, i_{m}: v_{1}\right)\right.\right.
$$

$$
\left.\left.\cdots \varphi_{n-1}^{m}\left(i_{1}, \cdots, i_{m}: v_{n-1}\right) \varphi_{n}^{m}\left(i_{1}, \cdots, i_{m}: \eta\right)\right]\left(v_{n}\right)\right\}\left(v_{n-1}\right) \text {, }
$$

where we use the notations $\left(U_{x} f\right)(y)$ and $\left(U_{x}^{*} f\right)(y)$ in place of (a), (b) in $\S 1$. We wish to prove that

$$
\begin{aligned}
U_{\eta}\left[\psi(\eta) \sum_{m=1}^{\infty} \sum_{i_{1}=1}^{n} \cdots \sum_{i_{m}=1}^{n} \varphi_{1}^{m}\left(i_{1}, \cdots, i_{m}: v_{1}\right) \cdots\right. \\
\left.\varphi_{n-1}^{m}\left(i_{1}, \cdots, i_{m}: v_{n-1}\right) \varphi_{n}^{m}\left(i_{1}, \cdots, i_{m}: \eta\right)\right]\left(v_{n}\right)
\end{aligned}
$$

is $L_{2}$-integrable with respect to $v_{n}$ for almost every $v_{1}, \cdots, v_{n-1}$, that is, (4.8) is well-defined. 
Let

$$
\psi_{N}(x)=\left\{\begin{array}{ccc}
\psi(x) & \text { if } & |x| \leqq N \\
0 & \text { if } & |x|>N
\end{array}\right.
$$

Since

$$
\begin{aligned}
\psi_{N}(\eta) & \sum_{m=1}^{\infty} \sum_{i_{1}=1}^{n} \cdots \sum_{i_{m}=1}^{n} \varphi_{1}^{m}\left(i_{1}, \cdots, i_{m}: v_{1}\right) \cdots \varphi_{n-1}^{m}\left(i_{1}, \cdots, i_{m}: v_{n-1}\right) \\
\cdot & \varphi_{n}^{m}\left(i_{1}, \cdots, i_{m}: \eta\right)
\end{aligned}
$$

is $L_{1}$-integrable with respect to $\eta$ by (4.5) for almost all $v_{1}, \cdots, v_{n-1}$, we have for each $v_{n}$ and for almost every $v_{1}, \cdots, v_{n-1}$

$$
\begin{aligned}
Q_{N}\left(v_{n}\right)= & U_{\eta}\left[\psi_{N}(\eta) \sum_{m=1}^{\infty} \sum_{i_{1}=1}^{n} \cdots \sum_{i_{m}=1}^{n} \varphi_{1}^{m}\left(i_{1}, \cdots, i_{m}: v_{1}\right)\right. \\
& \left.\cdots \varphi_{n-1}^{m}\left(i_{1}, \cdots, i_{m}: v_{n-1}\right) \varphi_{n}^{m}\left(i_{1}, \cdots, i_{m}: \eta\right)\right]\left(v_{n}\right) \\
= & \sum_{m=1}^{\infty} \sum_{i_{1}=1}^{n} \cdots \sum_{i_{m}=1}^{n} \varphi_{1}^{m}\left(i_{1}, \cdots, i_{m}: v_{1}\right) \cdots \\
& \cdot \varphi_{n-1}^{m}\left(i_{1}, \cdots, i_{m}: v_{n-1}\right) U_{\eta}\left[\psi_{N}(\eta) \varphi_{n}^{m}\left(i_{1}, \cdots, i_{m}: \eta\right)\right]\left(v_{n}\right)
\end{aligned}
$$

From (4.4), it follows that for almost all $v_{1}, \cdots, v_{n-1}$

$$
\begin{gathered}
\sum_{m=1}^{\infty} \sum_{i_{1}=1}^{n} \cdots \sum_{i_{m}=1}^{n}\left|\varphi_{1}^{m}\left(i_{1}, \cdots, i_{m}: v_{1}\right) \cdots \varphi_{n-1}^{m}\left(i_{1}, \cdots, i_{m}: v_{n-1}\right)\right| \\
\left\|\varphi_{n}^{m}\left(i_{1}, \cdots, i_{m}: \cdot\right)\right\|_{\infty} \cdot\left\|\psi_{N}\right\| \leqq\left\|\psi_{N}\right\| \sum_{m=1}^{\infty} N_{0}\left(F_{m}\right),
\end{gathered}
$$

where $\|\cdot\|_{\infty}$ means the essential supremum norm.

By using (4.10) we can prove that the last member of (4.9) also converges in the $L_{2}$-norm topology with respect to $v_{n}$ for almost every $v_{1}, \cdots, v_{n-1}$. By [13. 12.53. Example (iii)], both limits with the infinite sum of the last member of (4.9) are equal to each other except on a null set. Therefore we see that $Q_{N}\left(v_{n}\right)$ is $L_{2}$-integrable with respect to $v_{n}$ for almost every $v_{1}, \cdots, v_{n-1}$. Next we wish to prove that $Q_{N}\left(v_{n}\right)$ converges to a $L_{2}$-integrable function $Q\left(v_{n}\right)$ in the $L_{2}$-norm topology with respect to $v_{n}$ as $N \rightarrow \infty$ for almost all $v_{1}, \cdots, v_{n-1}$. From (4.4), it follows that

$$
\begin{aligned}
& \| \sum_{m=1}^{\infty} \sum_{i_{1}=1}^{n} \cdots \sum_{i_{m}=1}^{n} \varphi_{1}^{m}\left(i_{1}, \cdots, i_{m}: v_{1}\right) \cdots \varphi_{n-1}^{m}\left(i_{1}, \cdots, i_{m}: v_{n-1}\right) \\
& \cdot\left\{U_{\eta}\left[\psi_{N}(\eta) \varphi_{n}^{m}\left(i_{1}, \cdots, i_{m}: \eta\right)\right](\cdot)\right. \\
& \left.\quad-U_{\eta}\left[\psi(\eta) \varphi_{n}^{m}\left(i_{1}, \cdots, i_{m}: \eta\right)\right](\cdot)\right\} \| \\
& \leqq\left\|\psi_{N}-\psi\right\| \sum_{m=1}^{\infty} N_{0}\left(F_{m}\right) \rightarrow 0 \text { as } N \rightarrow \infty
\end{aligned}
$$


for almost all $v_{1}, \cdots, v_{n-1}$, where the infinite sum is taken in the $L_{2}$-norm topology. Hence we obtain from (4.11) that

$$
\begin{aligned}
\operatorname{lij.m} & Q_{N}\left(v_{n}\right) \\
= & 1 . i_{N \rightarrow \infty} \sum_{m=1}^{\infty} \sum_{i_{1}=1}^{n} \cdots \sum_{i_{m}=1}^{n} \varphi_{1}^{m}\left(i_{1}, \cdots, i_{m}: v_{1}\right) \cdots \\
& \cdot \varphi_{n-1}^{m}\left(i_{1}, \cdots, i_{m}: v_{n-1}\right) U_{\eta}\left[\psi_{N}(\eta) \varphi_{n}^{m}\left(i_{1}, \cdots, i_{m}: \eta\right)\right]^{\prime}\left(v_{n}\right) \\
= & \sum_{m=1}^{\infty} \sum_{i_{1}=1}^{n} \cdots \sum_{i_{m}=1}^{n} \varphi_{1}^{m}\left(i_{1}, \cdots, i_{m}: v_{1}\right) \cdots \\
& \cdot \varphi_{n-1}^{m}\left(i_{1}, \cdots, i_{m}: v_{n-1}\right) U_{\eta}\left[\psi(\eta) \varphi_{n}^{m}\left(i_{1}, \cdots, i_{m}: \eta\right)\right]\left(v_{n}\right) \\
= & Q\left(v_{n}\right)
\end{aligned}
$$

for almost every $v_{1}, \cdots, v_{n-1}$. Here the outer sum in the third member converges in the $L_{2}$-norm topology. Therefore we obtain the facts that (4.8) is $L_{2}$-integrable with respect to $v_{n}$ for almost every $v_{1}, \cdots, v_{n-1}$ and in the sense of $L_{2}$-equivalence

$$
\begin{aligned}
Q(\cdot)= & U_{\eta}\left[\psi(\eta) \sum_{m=1}^{\infty} \sum_{i_{1}=1}^{n} \cdots \sum_{i_{m}=1}^{n} \varphi_{1}^{m}\left(i_{1}, \cdots, i_{m}: v_{1}\right) \cdots\right. \\
& \left.\cdot \varphi_{n-1}^{m}\left(i_{1}, \cdots, i_{m}: v_{n-1}\right) \varphi_{n}^{m}\left(i_{1}, \cdots, i_{m}: \eta\right)\right](\cdot)
\end{aligned}
$$

for almost every $v_{1}, \cdots, v_{n-1}$.

Next we wish to prove that (4.7) is $L_{2}$-integrable with respect to $v_{n-1}$ for almost all $v_{1}, \cdots, v_{n-2}$.

Let

$$
X_{N}(x)=\left\{\begin{array}{lll}
1 & \text { if } & |x| \leqq N \\
0 & \text { if } & |x|>N
\end{array}\right.
$$

From (4.12) and from the fact that $Q$ is in $L_{2}$, it follows that

$$
\begin{aligned}
& X_{N}\left(v_{n}\right) Q\left(v_{n}\right) \exp \left(-\frac{b-t_{n-1}}{\lambda}\left|v_{n}\right|^{\alpha}\right) \\
& =X_{N}\left(v_{n}\right) \exp \left(-\frac{b-t_{n-1}}{\lambda}\left|v_{n}\right|^{\alpha}\right) \sum_{m=1}^{\infty} \sum_{n_{1}=1}^{n} \cdots \sum_{i_{m}=1}^{n} \varphi_{1}^{m}\left(i_{1}, \cdots, i_{m}: v_{1}\right) \\
& \cdots \varphi_{n-1}^{m}\left(i_{1}, \cdots, i_{m}: v_{n-1}\right) U_{\eta}\left[\psi(\eta) \varphi_{n}^{m}\left(i_{1}, \cdots, i_{m}: \eta\right)\right]\left(v_{n}\right)
\end{aligned}
$$

is $L_{1}$-integrable in $v_{n}$ for almost all $v_{1}, \cdots, v_{n-1}$. Since the infinite sum of the third member of (4.12) converges to $Q\left(v_{n}\right)$ in the $L_{2}$-norm topology, we have for almost all $v_{n}$ and for almost every $v_{1}, \cdots, v_{n-1}$, 


$$
\begin{aligned}
& X_{N}\left(v_{n}\right) Q\left(v_{n}\right) \exp \left(-\frac{b-t_{n-1}}{\lambda}\left|v_{n}\right|^{\alpha}\right) \\
& =\sum_{m=1}^{\infty} \sum_{i_{1}=1}^{n} \cdots \sum_{i_{m}=1}^{n} \varphi_{1}^{m}\left(i_{1}, \cdots, i_{m}: v_{1}\right) \cdots \varphi_{n-1}^{m}\left(i_{1}, \cdots, i_{m}: v_{n-1}\right) \\
& \quad \cdot X_{N}\left(v_{n}\right) \exp \left(-\frac{b-t_{n-1}}{\lambda}\left|v_{n}\right|^{\alpha}\right) U_{\eta}\left[\psi(\eta) \varphi_{n}^{m}\left(i_{1}, \cdots, i_{m}: \eta\right)\right]\left(v_{n}\right)
\end{aligned}
$$

where the infinite sum of the right side is taken in the $L_{2}$-norm topology. With the right side of (4.14), from the Schwartz inequality and (4.4), it follows that

$$
\begin{aligned}
& \sum_{m=1}^{\infty} \sum_{i_{1}=1}^{n} \cdots \sum_{i_{m}=1}^{n}\left|\varphi_{1}^{m}\left(i_{1}, \cdots, i_{m}: v_{1}\right) \cdots \varphi_{n-2}^{m}\left(i_{1}, \cdots, i_{m}: v_{n-2}\right)\right| \\
& \left\|\varphi_{n-1}^{m}\left(i_{1}, \cdots, i_{m}: \cdot\right)\right\|_{\infty} \\
& \quad \cdot\left\|X_{N} \exp \left(-\frac{b-t_{n-1}}{\lambda}|\cdot|^{\alpha}\right) U_{\eta}\left[\psi(\eta) \varphi_{n}^{m}\left(i_{1}, \cdots, i_{m}: \eta\right)\right](\cdot)\right\|_{1} \\
& \leqq(2 N)^{1 / 2}\|\psi\| \sum_{m=1}^{\infty} N_{0}\left(F_{m}\right)<\infty .
\end{aligned}
$$

From this fact and from [13. 10.83], it follows that for almost every $v_{1}, \cdots, v_{n-1}$, the infinite sum of the right side of (4.14) converges pointwisely for almost all $v_{n}$. Hence by [13. 12.53. Example (iii)], both limits with the infinite sum of the right side of (4.14) are equal to each other except on a null set. By the Dominated Convergence Theorem, we have

$$
\begin{aligned}
& (2 \pi)^{-1 / 2} \int_{-\infty}^{\infty} \exp \left(i v_{n-1} v_{n}\right) X_{N}\left(v_{n}\right) Q\left(v_{n}\right) \exp \left(-\frac{b-t_{n-1}}{\lambda}\left|v_{n}\right|^{\alpha}\right) d v_{n} \\
& =\lim _{k \rightarrow \infty}(2 \pi)^{-1 / 2} \int_{-\infty}^{\infty} \exp \left(i v_{n-1} v_{n}\right) \sum_{m=1}^{k} \sum_{i_{1}=1}^{n} \cdots \sum_{i_{m}=1}^{n} \varphi_{1}^{m}\left(i_{1}, \cdots, i_{m}: v_{1}\right) \\
& \quad \cdots \varphi_{n-1}^{m}\left(i_{1}, \cdots, i_{m}: v_{n-1}\right) X_{N}\left(v_{n}\right) \exp \left(-\frac{b-t_{n-1}}{\lambda}\left|v_{n}\right|^{\alpha}\right) \\
& \cdot U_{\eta}\left[\psi(\eta) \varphi_{n}^{m}\left(i_{1}, \cdots, i_{m}: \eta\right)\right]\left(v_{n}\right) d v_{n}
\end{aligned}
$$

$$
\begin{aligned}
= & \lim _{k \rightarrow \infty} \sum_{m=1}^{k} \sum_{i_{1}=1}^{n} \cdots \sum_{i_{m}=1}^{n} \varphi_{1}^{m}\left(i_{1}, \cdots, i_{m}: v_{1}\right) \cdots \\
& \cdot \varphi_{n-1}^{m}\left(i_{1}, \cdots, i_{m}: v_{n-1}\right) U_{v_{n}}^{*}\left\{X_{N}\left(v_{n}\right) \exp \left(-\frac{b-t_{n-1}}{\lambda}\left|v_{n}\right|^{\alpha}\right)\right. \\
& \left.\cdot U_{\eta}\left[\psi(\eta) \varphi_{n}^{m}\left(i_{1}, \cdots, i_{m}: \eta\right)\right]\left(v_{n}\right)\right\}\left(v_{n-1}\right)
\end{aligned}
$$




$$
\begin{aligned}
&= \sum_{m=1}^{\infty} \sum_{i_{1}=1}^{n} \cdots \sum_{i_{m}=1}^{n} \varphi_{1}^{m}\left(i_{1}, \cdots, i_{m}: v_{1}\right) \cdots \varphi_{n-1}^{m}\left(i_{1}, \cdots, i_{m}: v_{n-1}\right) \\
& \cdot U_{v_{n}}^{*}\left\{X_{N}\left(v_{n}\right) \exp \left(-\frac{b-t_{n-1}}{\lambda}\left|v_{n}\right|^{\alpha}\right) U_{\eta}\left[\psi(\eta) \varphi_{n}^{m}\left(i_{1}, \cdots, i_{m}: \eta\right)\right]\left(v_{n}\right)\right\} \\
& \times\left(v_{n-1}\right)
\end{aligned}
$$

in the sense of the pointwise convergence with respect to $v_{n-1}$ for almost every $v_{1}, \cdots, v_{n-2}$. From (4.4), it follows that for almost all $v_{1}, \cdots, v_{n-2}$

$$
\begin{aligned}
& \sum_{m=1}^{\infty} \sum_{i_{1}=1}^{n} \cdots \sum_{i_{m}=1}^{n}\left|\varphi_{1}^{m}\left(i_{1}, \cdots, i_{m}: v_{1}\right) \cdots \varphi_{n-2}^{m}\left(i_{1}, \cdots, i_{m}: v_{n-2}\right)\right| \\
& \left\|\varphi_{n-1}^{m}\left(i_{1}, \cdots, i_{m}: \cdot\right)\right\|_{\infty} \cdot \| U_{v_{n}}^{*}\left\{X_{N}\left(v_{n}\right) \exp \left(-\frac{b-t_{n-1}}{\lambda}\left|v_{n}\right|^{\alpha}\right)\right. \\
& \left.\cdot U_{\eta}\left[\psi(\eta) \varphi_{n}^{m}\left(i_{1}, \cdots, i_{m}: \eta\right)\right]\left(v_{n}\right)\right\}(\cdot)\left\|\leqq \sum_{m=1}^{\infty} N_{0}\left(F_{m}\right)\right\| \psi \| .
\end{aligned}
$$

By this fact, the last member of (4.15) also converges in the $L_{2}$-norm topology with respect to $v_{n-1}$ for almost all $v_{1}, \cdots, v_{n-2}$. From the fact that both limits of the last member of (4.15) are equal to each other except on a null set, by an argument similar to that used in obtaining (4.12), we can see that for almost every $v_{1}, \cdots, v_{n-2},(4.15)$ converges to

$$
\begin{aligned}
\sum_{m=1}^{\infty} \sum_{i_{1}=1}^{n} \cdots & \sum_{l_{m}=1}^{n} \varphi_{1}^{m}\left(i_{1}, \cdots, i_{m}: v_{1}\right) \cdots \\
\cdot & \varphi_{n-1}^{m}\left(i_{1}, \cdots, i_{m}: v_{n-1}\right) U_{v_{n}}^{*}\left\{\exp \left(-\frac{b-t_{n-1}}{\lambda}\left|v_{n}\right|^{\alpha}\right)\right. \\
\cdot & \left.U_{\eta}\left[\psi(\eta) \varphi_{n}^{m}\left(i_{1}, \cdots, i_{m}: \eta\right)\right]\left(v_{n}\right)\right\}\left(v_{n-1}\right)
\end{aligned}
$$

in the $L_{2}$-norm with respect to $v_{n-1}$, where the infinite sum of (4.16) is taken in the $L_{2}$-norm topology with respect to $v_{n-1}$. From the above argument, it follows that (4.6) is $L_{2}$-integrable in $v_{n-1}$ for almost every $v_{1}, \cdots, v_{n-2}$ and

$$
\begin{aligned}
(4.6)= & \sum_{m=1}^{\infty} \sum_{i_{1}=1}^{n} \cdots \sum_{i_{m}=1}^{n} \varphi_{1}^{m}\left(i_{1}, \cdots, i_{m}: v_{1}\right) \cdots \varphi_{n-1}^{m}\left(i_{1}, \cdots, i_{m}: v_{n-1}\right) \\
& \cdot \int_{-\infty}^{\infty} p_{\lambda}^{\alpha}\left(b-t_{n-1}, v_{n}-v_{n-1}\right) \psi\left(v_{n}\right) \varphi_{n}^{m}\left(i_{1}, \cdots, i_{m}: v_{n}\right) d v_{n}
\end{aligned}
$$

in the sense of the $L_{2}$-equivalence. By repeating the above argument, we can prove that

$$
\left(\mathscr{F}_{\lambda}^{\alpha, \sigma}(F) \psi\right)(\xi)=\sum_{m=1}^{\infty}\left(\mathscr{F}_{\lambda}^{\alpha, \sigma}\left(F_{m}\right) \psi\right)(\xi)
$$


where the sum is taken in the $L_{2}$-norm topology with respect to $\xi$. By using the fact that

$$
\sum_{m=1}^{\infty}\left\|\mathscr{F}^{\alpha, \sigma}\left(F_{m}\right)\right\| \leqq \sum_{m=1}^{\infty} N_{0}\left(F_{m}\right)<\infty
$$

we have that

$$
\begin{aligned}
\lim _{\|\sigma\| \rightarrow 0} \mathscr{F}_{\lambda}^{\alpha, \sigma}(F) \psi & =\lim _{\|\sigma\| \rightarrow 0} \lim _{N \rightarrow \infty} \sum_{m=1}^{N} \underset{\lambda}{\mathscr{F}_{\lambda}^{\alpha, \sigma}\left(F_{m}\right) \psi} \\
& =\lim _{N \rightarrow \infty} \lim _{\|\sigma\| \rightarrow 0} \sum_{m=1}^{N} \underset{\mathscr{F}_{\lambda}^{\alpha, \sigma}}{\mathscr{F}_{i}}\left(F_{m}\right) \psi=\lim _{N \rightarrow \infty} \sum_{m=1}^{N} \mathscr{F}_{\lambda}^{\alpha}\left(F_{m}\right) \psi \\
& =\sum_{m=1}^{\infty} \mathscr{F}_{\lambda}^{\alpha}\left(F_{m}\right) \psi
\end{aligned}
$$

in the $L_{2}$-norm. From (4.17), (4.2) follows.

Corollary 4.1. Let

$$
F(x)=\exp \left\{\int_{a}^{b} \theta(s, x(s)) d s\right\}
$$

where $\theta(s, u)$ is bounded by $M$ and measurable in $[a, b] \times R$. Then for $\lambda \in D$

$$
\mathscr{F}_{\lambda}^{\alpha}(F) \psi=\sum_{m=0}^{\infty} \mathscr{J}_{\lambda}^{\alpha}\left(F_{m}\right) \psi
$$

where $\psi \in L_{2}$ and

$$
F_{m}(x)=\left(\int_{a}^{b} \theta(s, x(s)) d s\right)^{m}
$$

and where the sum is taken in the norm topology. Furthermore

$$
\left\|\mathscr{F}_{\lambda}^{\alpha}(F)\right\| \leqq e^{(b-a) M} .
$$

Proof. By expanding $F(x)$ into a series of the functionals in $A_{0}$ and by applying Theorem 4.1 and Corollary 3.1 , we obtain Corollary.

5. Integral equation. Let $\theta(t, u)$ be a Borel function on $\left[0, t_{0}\right] \times R$ and bounded by $M$. For each $t \in\left(0, t_{0}\right]$, let $\theta_{t}(s, u)$ be defined on $[0, t] \times R$ by $\theta_{t}(s, u) \equiv \theta(t-s, u)$. In what follows we shall consider the functional 


$$
F^{t}(x)=\exp \left\{\int_{0}^{t} \theta_{t}(s, x(s)) d s\right\}
$$

For convenience, let us put

$$
\begin{aligned}
G(t, \xi, \lambda) & =\left(\mathscr{F}_{\lambda}^{\alpha}\left(F^{t}\right) \psi\right)(\xi), \\
g_{m}(t, \xi, \lambda) & =\left(\mathscr{J}_{\lambda}^{\alpha}\left(F_{m}^{t}\right) \psi\right)(\xi),
\end{aligned}
$$

where

$$
F_{m}^{t}(x)=\left\{\int_{0}^{t} \theta_{t}(s, x(s)) d s\right\}^{m}, \quad m=0,1, \cdots
$$

THEOREM 5.1. $G(t, \xi, \lambda)$ satisfies the integral equation

$$
G(t, \xi, \lambda)=\int_{-\infty}^{\infty} p_{\lambda}^{\alpha}(t, u-\xi) \psi(u) d u
$$

$$
+(B) \int_{0}^{t}\left[\int_{-\infty}^{\infty} p_{\lambda}^{\alpha}(t-s, u-\xi) \theta(s, u) G(s, u, \lambda) d u\right] d s
$$

where $t_{0} \geqq t>0, \lambda \in D$ and $\psi \in L_{2}$.

Proof. Let $\lambda \in D$ and $\psi \in L_{2}$. From Corollary 4.1, we have

$$
G(t, \xi, \lambda)=\sum_{m=0}^{\infty} g_{m}(t, \xi, \lambda)
$$

where the sum is taken in the $L_{2}$-norm topology.

At first we will prove that

$$
\sum_{m=0}^{\infty} g_{m}(t, \xi, \lambda)=\int_{-\infty}^{\infty} p_{\lambda}^{\alpha}(t, u-\xi) \psi(u) d u
$$

$$
+\sum_{m=0}^{\infty}(B) \int_{0}^{t} d s\left[\int_{-\infty}^{\infty} p_{\lambda}^{\alpha}(t-s, u-\xi) \theta(s, u) g_{m}(s, u, \lambda) d u\right]
$$

where both sums are taken in the $L_{2}$-norm topology. In order to prove this, since

$$
g_{0}(t, \xi, \lambda)=\int_{-\infty}^{\infty}, p_{\lambda}^{\alpha}(t, u-\xi) \psi(u) d u
$$

it suffices to prove that 


$$
g_{m+1}(t, \xi, \lambda)=(B) \int_{0}^{t} d s\left[\int_{-\infty}^{\infty} p_{\lambda}^{\alpha}(t-s, u-\xi) \theta(s, u) g_{m}(s, u, \lambda) d u\right]
$$

We prove that the integrand of (5.3) is Bochner integrable with respect to the variable $s$ over $[0, t]$. By [5. Theorem 3.7.12 and the remark following], it holds that

$$
\begin{aligned}
Y(s, \xi)= & \int_{-\infty}^{\infty} p_{\lambda}^{\alpha}(t-s, u-\xi) \theta(s, u) g_{m}(s, u, \lambda) d u \\
= & (B) \int_{S_{m}(0, s)}(m) \int d s_{1} \cdots d s_{m}\left\{\int_{-\infty}^{\infty} p_{\lambda}^{\alpha}(t-s, u-\xi) \theta(s, u) d u\right. \\
& \cdot \int_{-\infty}^{\infty} p_{\lambda}^{\alpha}\left(s_{1}, u_{1}-u\right) \theta_{s}\left(s_{1}, u_{1}\right) d u_{1} \cdots \int_{-\infty}^{\infty} p_{\lambda}^{\alpha}\left(s_{m}-s_{m-1}, u_{m}-u_{m-1}\right) \\
& \left.\cdot \theta_{s}\left(s_{m}, u_{m}\right) d u_{m} \int_{-\infty}^{\infty} p_{\lambda}^{\alpha}\left(s-s_{m}, u_{m+1}-u_{m}\right) \psi\left(u_{m+1}\right) d u_{m+1}\right\}
\end{aligned}
$$

for almost all $s \in[0, t]$ and hence by using [5. Theorem 3.7.6], we obtain for almost all $s \in[0, t]$,

(a) $\|Y\| \leqq M^{m+1} \frac{t^{m}}{m !}\|\psi\|$.

Furthermore it is necessary to show that $Y$ is strongly measurable in $s$. To show this, it is sufficient to show that $Y$ is weakly measurable in $s$ since $L_{2}$ is separable. Let $\varphi$ be in $L_{2}$. By a change of variables, we have

$$
\begin{gathered}
\int_{s_{m}(0, s)}(m) \int d s_{1} \cdots d s_{m}\left\{\int _ { - \infty } ^ { \infty } \overline { \varphi ( \xi ) } \left[\int_{-\infty}^{\infty} p_{\lambda}^{\alpha}(t-s, u-\xi) \theta(s, u) d u\right.\right. \\
\cdot \int_{-\infty}^{\infty} p_{\lambda}^{\alpha}\left(s_{1}, u_{1}-u\right) \theta_{s}\left(s_{1}, u_{1}\right) d u_{1} \cdots \int_{-\infty}^{\infty} p_{\lambda}^{\alpha}\left(s_{m}-s_{m-1}, u_{m}-u_{m-1}\right) \\
\left.\left.\cdot \theta_{s}\left(s_{m}, u_{m}\right) d u_{m} \int_{-\infty}^{\infty} p_{\lambda}^{\alpha}\left(s-s_{m}, u_{m+1}-u_{m}\right) \psi\left(u_{m+1}\right) d u_{m+1}\right] d \xi\right\}
\end{gathered}
$$

$$
\begin{aligned}
= & \int_{S_{m}\left(\tau_{1}, t\right)}(m) \int d \tau_{2} \cdots d \tau_{m+1}\left\{\int _ { - \infty } ^ { \infty } \overline { \varphi ( \xi ) } \left[\int_{-\infty}^{\infty} p_{\lambda}^{\alpha}\left(\tau_{1}, u-\xi\right) \theta_{t}\left(\tau_{1}, u\right) d u\right.\right. \\
& \cdot \int_{-\infty}^{\infty} p_{\lambda}^{\alpha}\left(\tau_{2}-\tau_{1}, u_{1}-u\right) \theta_{t}\left(\tau_{2}, u_{1}\right) d u_{1} \cdots \int_{-\infty}^{\infty} p_{\lambda}^{\alpha}\left(\tau_{m+1}-\tau_{m}, u_{m}-u_{m-1}\right) \\
& \left.\left.\cdot \theta_{t}\left(\tau_{m+1}, u_{m}\right) d u_{m} \int_{-\infty}^{\infty} p_{\lambda}^{\alpha}\left(t-\tau_{m+1}, u_{m+1}-u_{m}\right) \psi\left(u_{m+1}\right) d u_{m+1}\right] d \xi\right\}
\end{aligned}
$$


where let $t-s=\tau_{1}, s_{1}=\tau_{2}-\tau_{1}, s_{2}=\tau_{3}-\tau_{1}, \cdots, s_{m}=\tau_{m+1}-\tau_{1}$. The integrand with the variables $\tau_{1}, \tau_{2}, \cdots, \tau_{m+1}$ of the right side of (5.4) is measurable in $\left(\tau_{1}, \cdots, \tau_{m+1}\right)$ on $S_{m+1}(0, t)$ as shown in the proof of Theorem 3.1 and integrable with respect to $\tau_{1}, \cdots, \tau_{m+1}$ over $S_{m+1}(0, t)$. Therefore by the Fubini Theorem, the right side of $(5.4)$ is measurable in $\tau_{1}$, hence we can say that

(b) $Y(s, \xi)$ is strongly measurable in $s$.

From (a) and (b), it follows that

(c) $Y(s, \xi)$ is Bochner integrable with respect to $s$ over $[0, t]$.

Therefore by [5. Theorem 3.7.12 and the remark following] and the Fubini Theorem,

$$
\begin{aligned}
\int_{-\infty}^{\infty} & \overline{\varphi(\xi)}\left\{(B) \int_{0}^{t} d s\left[\int_{-\infty}^{\infty} p_{\lambda}^{\alpha}(t-s, u-\xi) \theta(s, u) g_{m}(s, u, \lambda) d u\right]\right\} d \xi \\
= & \int_{0}^{t} d s \int_{-\infty}^{\infty} \overline{\varphi(\xi)}\left[\int_{-\infty}^{\infty} p_{\lambda}^{\alpha}(t-s, u-\xi) \theta(s, u) g_{m}(s, u, \lambda) d u\right] d \xi \\
= & \int_{0}^{t} d s\left\{\int _ { S _ { m } ( 0 , s ) } ( m ) \int d s _ { 1 } \cdots d s _ { m } \left[\int _ { - \infty } ^ { \infty } \overline { \varphi ( \xi ) } \left[\int_{-\infty}^{\infty} p_{\lambda}^{\alpha}(t-s, u\right.\right.\right. \\
& \cdot \int_{-\infty}^{\infty} p_{\lambda}^{\alpha}\left(s_{1}, u_{1}-u\right) \theta_{s}\left(s_{1}, u_{1}\right) d u_{1} \cdots \\
& \left.\left.\left.\cdot \int_{-\infty}^{\infty} p_{\lambda}^{\alpha}\left(s-s_{m}, u_{m+1}-u_{m}\right) \psi\left(u_{m+1}\right) d u_{m+1}\right] d \xi\right]\right\} \\
= & \int_{S_{m+1}(0, t)}^{(m+1)} d d \tau_{1} \cdots d \tau_{m+1}\left\{\int _ { - \infty } ^ { \infty } \overline { \varphi ( \xi ) } \left[\int _ { - \infty } ^ { \infty } p _ { \lambda } ^ { \alpha } \left(\tau_{1}, u\right.\right.\right. \\
& \cdot \int_{-\infty}^{\infty} p_{\lambda}^{\alpha}\left(\tau_{2}-\tau_{1}, u_{1}-u\right) \theta_{t}\left(\tau_{2}, u_{1}\right) d u_{1} \cdots \\
& \left.\left.\cdot \int_{-\infty}^{\infty} p_{\lambda}^{\alpha}\left(t-\tau_{m+1}, u_{m+1}-u_{m}\right) \psi\left(u_{m+1}\right) d u_{m+1}\right] d \xi\right\} \\
= & \int_{-\infty}^{\infty} \frac{\varphi(\xi)}{\varphi(\xi)} g_{m+1}(t, \xi, \lambda) d \xi .
\end{aligned}
$$

From this fact, (5.3) follows. Therefore (5.2) is valid. 
From Corollary 4.1, Theorem 1.1 and from the boundedness of $\theta$, we obtain that

$$
\begin{array}{r}
\sum_{m=0}^{n} \int_{-\infty}^{\infty} p_{\lambda}^{\alpha}(t-s, u-\xi) \theta(s, u) g_{m}(s, u, \lambda) d u \\
\quad \rightarrow \int_{-\infty}^{\infty} p_{\lambda}^{\alpha}(t-s, u-\xi) \theta(s, u) G(s, u, \lambda) d u
\end{array}
$$

in the $L_{2}$-norm topology as $n \rightarrow \infty$ for almost all $s \in[0, t]$. Since the left side of (5.5) is also Bochner integrable in $s$ over $[0, t]$ by (c), it follows from (a) and [5. Theorem 3.7.9] that the right side of (5.5) is Bochner integrable in $s$ over $[0, t]$. Furthermore, we have

$$
\begin{aligned}
\sum_{m=0}^{\infty}( & B) \int_{0}^{t} d s \int_{-\infty}^{\infty} p_{\lambda}^{\alpha}(t-s, u-\xi) \theta(s, u) g_{m}(s, u, \lambda) d u \\
= & \lim _{n \rightarrow \infty}(B) \int_{0}^{t} d s\left[\sum_{m=0}^{n} \int_{-\infty}^{\infty} p_{\lambda}^{\alpha}(t-s, u-\xi) \theta(s, u) g_{m}(s, u, \lambda) d u\right] \\
= & (B) \int_{0}^{t} d s\left[\sum_{m=0}^{\infty} \int_{-\infty}^{\infty} p_{\lambda}^{\alpha}(t-s, u-\xi) \theta(s, u) g_{m}(s, u, \lambda) d u\right] \\
= & (B) \int_{0}^{t} d s\left[\int_{-\infty}^{\infty} p_{\lambda}^{\alpha}(t-s, u-\xi) \theta(s, u) G(s, u, \lambda) d u\right]
\end{aligned}
$$

where both infinite sums are to be taken in the sense of the norm topology. By this fact, we see that $G(t, \xi, \lambda)$ is a solution of the integral equation (5.1).

\section{REFERENCES}

1. R. H. Cameron and D. A. Storvick, An operator valued function space integral and a related integral equation, J. Math. Mech., 18 (1968), 517-552.

2 - An integral equation related to the Schroedinger equation with an application to integration in function space, in "Problems in Analysis", Prineeton Univ. Press, Princeton, New Jersey, (1970).

3. - An operator valued function space integral applied to multiple integrals of functions of class $L_{1}$, Nagoya Math. J., 51 (1973), 91-122.

4. —, Two related integrals over spaces of continuous functions, Pacific J. Math., 55 (1974), 19-37.

5. E. Hille and R. S. Phillips, Functional analysis and semigroups, Amer. Math. Soc. Colloq. Publ., 31 (1957).

6. G. W. Johnson and D. L. Skoug, Operator-valued Feynman integrals of certain finite-dimensional functionals, Proc. Amer. Math. Soc., 24 (1970), 774-780.

7. — An operator valued function space integral: a sequel to Cameron and Storvick's paper, Proc. Amer. Soc., 27 (1971), 514-519.

8. - Feynman integrals of non-factorable finite-dimensional functionals, Pacific J. Math., 45 (1973), 257-267. 
9. - A Banach algebra of Feynman integrable functionals with application to an integral equation formally equivalent to Schroedinger's equation, J. Functional Analysis, 12 (1973), 129-152.

10. - Cameron and Storvick's function space integral for certain Banach spaces of functionals, J. London Math. Soc., (2), 9 (1974), 103-117.

11. E. Nelson, Feynman integrals and the Schroedinger equation, J. Math. Phys., 5 (1964), 332-343.

12. A. Renyi, Probability theory, North-Holland Publishing Company, (1970).

13. E. C. Titchmarsh, The Theory of Functions, Oxford Univ. Press, Second Edition, (1948).

14. A. Zygmund, Trigonometric Series, vol. II, Second Edition Cambridge Univ. Press, (1959).

Received July 18, 1975 and in revised form May 12, 1976. The author is grateful to the referee for his many helpful suggestions.

IBARAKI UNIVERSITY 



\section{PACIFIC JOURNAL OF MATHEMATICS}

\section{EDITORS}

RICHARI) ARENS (Managing Editor)

University of California

Los Angeles, CA 90024

R. A. Beaumiont

University of Washington

Seattle, WA 98105

\section{J. DugunduI}

Department of Mathematics University of Southern California Los Angeles, CA 90007

D. Gilbarg and J. Milgram Stanford University

Stanford, CA 94305

\section{ASSOCIATE EDITORS}

E. F. BECKENBACH
B. H. NEUMANN

F. Wolf

K. YoshidA

\section{SUPPORTING INSTITUTIONS}

UNIVERSITY OF BRITISH COLUMBIA CALIFORNIA INSTITUTE OF TECHNOLOGY

UNIVERSITY OF CALIFORNIA

MONTANA STATE UNIVERSITY

UNIVERSITY OF NEVADA

NEW MEXICO STATE UNIVERSITY

OREGON STATE UNIVERSITY

UNIVERSITY OF OREGON

OSAKA UNIVERSITY

\author{
UNIVERSITY OF SOUTHERN CALIFORNIA \\ STANFORD UNIVERSITY \\ UNIVERSITY OF HAWAII \\ UNIVERSITY OF TOKYO \\ UNIVERSITY OF UTAH \\ WASHINGTON STATE UNIVERSITY \\ UNIVERSITY OF WASHINGTON \\ AMERICAN MATHEMATICAL SOCIETY
}

The Supporting Institutions listed above contribute to the cost of publication of this Journal, but they are not owners or publishers and have no responsibility for its contents or policies.

Mathematical papers intended for publication in the Pacific Journal of Mathematics should be in typed form or offset-reproduced (not dittoed), double spaced with large margins. Underline Greek letters in red, German in green, and script in blue. The first $p: 1<$ graph or two must be capable of being used separately as a synopsis of the entire paper. Items of the biblography should not be cited there unless absolutely necessary, in which case they must he identified by author and Journal, rather than by item number. Manuscripts, in duplicate, may be sent to any one of the four editors. Please classify according to the scheme of Math. Reviews, Index to Vol. 39. All other communications should be addressed to the managing editor, or Elaine Barth, University of California, Los Angeles, California, 90024.

100 reprints are provided free for each article, only if page charges have been substantially paid. Additional copies may be obtained at cost in multiples of 50 .

The Pacific Journal of Mathematics is issued monthly as of January 1966. Regular subscription rate: $\$ 72.00$ a year (6 Vols., 12 issues). Special rate: $\$ 36.00$ a year to individual members of supporting institutions.

Subscriptions, orders for back numbers, and changes of address should be sent to Pacific Journal of Mathematics, 103 Highland Boulevard, Berkeley, California, 94708.

PUBLISHED BY PACIFIC JOURNAL OF MATHEMATICS, A NON-PROFIT CORPORATION Printed at Jerusalem Academic Press, POB 2390, Jerusalem, Israel.

\section{Copyright (C) 1976 Pacific Journal of Mathematics} All Rights Reserved 


\section{Pacific Journal of Mathematics}

\section{Vol. 66, No. 2 December, 1976}

Gerald A. Beer, Tax structures whose progressivity is inflation neutral..... 305

William M. Cornette, A generalization of the unit interval............. 313

David E. Evans, Unbounded completely positive linear maps on

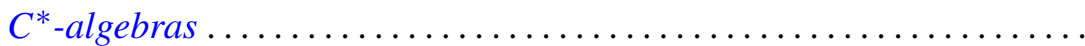

Hector O. Fattorini, Some remarks on convolution equations for

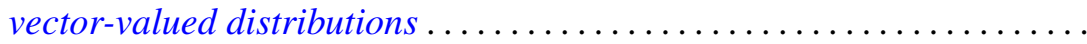

Amassa Courtney Fauntleroy, Automorphism groups of unipotent groups of

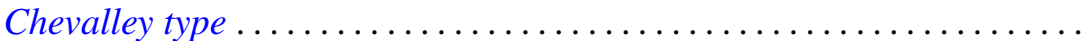

Christian C. Fenske and Heinz-Otto Peitgen, On fixed points of zero index in

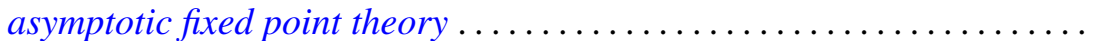

Atsushi Inoue, On a class of unbounded operator algebras. II ............

Herbert Meyer Kamowitz, The spectra of endomorphisms of algebras of

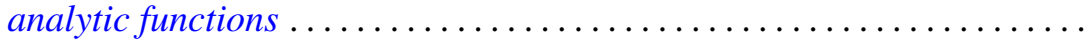

Jimmie Don Lawson, Embeddings of compact convex sets and locally compact cones ....................................

William Lindgren and Peter Joseph Nyikos, Spaces with bases satisfying certain order and intersection properties .....................

Emily Mann Peck, Lattice projections on continuous function spaces ...... 477

Morris Marden and Peter A. McCoy, Level sets of polynomials in $n$ real variables...

Francis Joseph Narcowich, An imbedding theorem for indeterminate Hermitian moment sequences......................

John Dacey O'Neill, Rings whose additive subgroups are subrings ...

Chull Park and David Lee Skoug, Wiener integrals over the sets bounded by sectionally continuous barriers .....................

Vladimir Scheffer, Partial regularity of solutions to the Navier-Stokes equations.

Eugene Spiegel and Allan Trojan, On semi-simple group algebras. II

Katsuo Takano, On Cameron and Storvick's operator valued function space integral 